\title{
KIRCHHOFF ELASTIC RODS IN THE THREE-SPHERE
}

\author{
SATOSHI KAWAKUBO
}

(Received September 17, 2002, revised January 15, 2004)

\begin{abstract}
The Kirchhoff elastic rod is one of the mathematical models of thin elastic rods, and is a critical point of the energy functional with the effect of bending and twisting. In this paper, we study Kirchhoff elastic rods in the three-sphere of constant curvature. In particular, we give explicit expressions of Kirchhoff elastic rods in terms of elliptic functions and integrals. In addition, we obtain equivalent conditions for Kirchhoff elastic rods to be closed, and give an example of closed Kirchhoff elastic rods.
\end{abstract}

1. Introduction. The mathematical models of thin elastic rods have been studied since the days of Daniel Bernoulli and Leonhard Euler. One of the famous models is Euler's elastica, which is a critical curve of the bending energy. The uniform symmetric case of Kirchhoff elastic rods is a generalization of the elastica and is the simplest model with the effect of bending and twisting.

Elasticae in Riemannian manifolds, except the Euclidean space, have been studied by many authors in [1], [2], [4], [8], [13], [16], [17], [18], [21], etc. One of the motivations of these papers is to investigate Willmore surfaces. Meanwhile, Kirchhoff elastic rods in Riemannian manifolds, except the Euclidean space, are studied in [19], [10]. Langer and Singer ([19]) derived the Hamiltonian systems associated to a class of variational problems, including the Kirchhoff elastic rod in 3-dimensional space forms, $\boldsymbol{R}^{3}, S^{3}, H^{3}$, and proved their Liouville integrability.

In the case of the Euclidean 3 -space $\boldsymbol{R}^{3}$, it is known that Kirchhoff elastic rods are explicitly expressed in cylindrical coordinates by Jacobi elliptic functions and integrals ([20], [22]). By using these explicit expressions, Ivey and Singer ([7]) completely classified the closed Kirchhoff elastic rods in $\boldsymbol{R}^{3}$ and determined their knot types.

In this paper, we study Kirchhoff elastic rods in the 3 -sphere $S^{3}$ of constant sectional curvature. We give explicit expressions of Kirchhoff elastic rods in $S^{3}$ in terms of Jacobi elliptic functions and integrals, and obtain the equivalent conditions for Kirchhoff elastic rods to be closed. Also, by using the closure conditions, we construct a smooth two-parameter family of closed Kirchhoff elastic rods, including both helices and non-helices.

Let $\mathcal{M}$ be an $n$-dimensional Riemannian manifold. Let $\gamma=\gamma(t):[0, l] \rightarrow \mathcal{M}$ be a smooth unit-speed curve, and $M(t)=\left(M_{1}(t), M_{2}(t), \ldots, M_{n-1}(t)\right)$ a smooth orthonormal

2000 Mathematics Subject Classification. Primary 58E10; Secondary 74K10, 74G05.

Key words and phrases. Elastic rods, calculus of variations.

Partly supported by the Grant-in-Aid for JSPS Fellows, The Ministry of Education, Culture, Sports, Science and Technology, Japan. 
frame field in the normal bundle along $\gamma$. We consider the pair $\{\gamma, M\}$ of $\gamma$ and $M$. In this paper, we call such a pair $\{\gamma, M\}$ a curve with adapted orthonormal frame, and $\gamma$ the centerline of it. The frame field $M$ describes the twisting of the material about the centerline $\gamma$. We note that for each $t,\left(\gamma^{\prime}(t), M_{1}(t), \ldots, M_{n-1}(t)\right)$ is an orthonormal basis of the tangent space to $\mathcal{M}$ at $\gamma(t)$. Let $v$ be a positive constant, which is determined by the material of the elastic rod. We define the energy $\mathfrak{T}$ as follows:

$$
\mathfrak{T}(\{\gamma, M\})=\int_{0}^{l}\left|\nabla_{t} \gamma^{\prime}\right|^{2} d t+v \sum_{i=1}^{n-1} \int_{0}^{l}\left|\nabla_{t}^{\perp} M_{i}\right|^{2} d t .
$$

Here, the first term of the right hand side is the bending (or elastic) energy of $\gamma$, and the second term is the energy representing the effect of twisting.

In Section 2, we calculate the first variation formula for $\mathfrak{T}$ and derive the Euler-Lagrange equation. We call a solution $\{\gamma, M\}$ of the Euler-Lagrange equation a Kirchhoff elastic rod. In Section 3, we give explicit expressions of the curvature and torsion of the centerline of a Kirchhoff elastic rod in $\mathcal{M}=\boldsymbol{R}^{3}, S^{3}, H^{3}$ of constant sectional curvature $G$, and then parametrize the space of the congruent classes of Kirchhoff elastic rods by four real numbers (Proposition 3.1). In the case of $\mathcal{M}=S^{3}$, we introduce another parametrization of the space by four real numbers, which we will write as $\beta, \eta, p, w$.

Let $\mathcal{M}=S^{3}$ and $\{\gamma, M\}$ a Kirchhoff elastic $\operatorname{rod}$ in $\mathcal{M}$. Let $\iota: S^{3} \rightarrow \boldsymbol{R}^{4}$ be an isometric embedding of $S^{3}$ into $\boldsymbol{R}^{4}$, with the canonical coordinates $\left(x_{1}, x_{2}, x_{3}, x_{4}\right)$, as the standard sphere of radius $1 / \sqrt{G}$. For such an embedding $\iota$, we take a local coordinate system $(r, \theta, \psi)$ in $S^{3}$ satisfying the following relations:

$$
x_{1}=r \cos \theta, \quad x_{2}=r \sin \theta, \quad x_{3}=\bar{r} \cos \psi, \quad x_{4}=\bar{r} \sin \psi,
$$

where $r>0$ and $\bar{r}=\sqrt{(1 / G)-r^{2}}$. The coordinate system $(r, \theta, \psi)$ is analogous to the system of cylindrical coordinates in $\boldsymbol{R}^{3}$, and each level surface of the coordinate $r$ is a Clifford torus instead of a cylinder.

To obtain the explicit expression for $\gamma$, we use the method of Killing vector fields used by Langer and Singer ([15], [17], [20], [7]). In Section 4, we construct some Killing vector fields associated to the Kirchhoff elastic $\operatorname{rod}\{\gamma, M\}$. In Section 5, by using these Killing vector fields, we give an appropriate orthogonal transformation $P$ on $\boldsymbol{R}^{4}$ and take the coordinates $(r, \theta, \psi)$ with respect to the embedding $P \circ \iota: S^{3} \rightarrow \boldsymbol{R}^{4}$ instead of $\iota$.

In Section 6, we give the explicit expressions of $r, \theta, \psi$ components of the centerline $\gamma$ in terms of Jacobi sn function and the incomplete elliptic integral of the third kind.

THEOREM 1.1 (cf. Theorem 6.1). Let $\{\gamma, M\}$ be a Kirchhoff elastic rod in $S^{3}$. Then there exists an orthogonal transformation $P$ on $\boldsymbol{R}^{4}$ satisfying the following: Let $(r, \theta, \psi)$ denote the coordinates as above with respect to the isometric embedding $P \circ \iota: S^{3} \rightarrow \boldsymbol{R}^{4}$, and $r(t), \theta(t), \psi(t)$ denote the $r, \theta, \psi$ components of $\gamma$. Then,

$$
r(t)=\sqrt{c_{1} \mathrm{sn}^{2}\left(c_{2} t, c_{3}\right)+c_{4}} .
$$


Moreover, if there exist no points where $r(t)=0$ or $1 / \sqrt{G}$ (, a condition being satisfied by generic Kirchhoff elastic rods), then

$$
\begin{aligned}
\theta(t) & =c_{5} t+c_{6} \Pi\left(c_{2} t, c_{7}, c_{3}\right), \\
\psi(t) & =c_{8} t+c_{9} \Pi\left(c_{2} t, c_{10}, c_{3}\right),
\end{aligned}
$$

where sn and $\Pi$ denote Jacobi sn function and the incomplete elliptic integral of the third kind, respectively. Also, $c_{1}, \ldots, c_{10}$ are real constants, which are explicitly expressed by the parameter $(\beta, \eta, p, w)$ representing the congruent class of $\{\gamma, M\}$. Even when there exists a point where $r(t)=0$ or $1 / \sqrt{G}$, the explicit formulas of $\theta(t), \psi(t)$ are obtained in the same way as above.

Here, we note that the elliptic modulus $c_{3}$ satisfies $0 \leqslant c_{3} \leqslant 1$. (In practice, $c_{3}=p$ holds. See below Section 3.) When $c_{3}=1$, the shape of $\gamma$ is quite different from the other cases. The case of $c_{3}=1$ is called the Hasimoto soliton case (see the last paragraph of Section 4). Also, when $c_{3}=0$, the centerline $\gamma$ is a helix. Except these two special cases (the helix case and the Hasimoto soliton case), $r(t), \theta^{\prime}(t), \psi^{\prime}(t)$ are periodic functions with the same primitive period. Let $\Delta \theta, \Delta \psi$ denote respectively the changes in $\theta(t), \psi(t)$ through the primitive period of $r$.

In Section 7 , we express $\Delta \theta, \Delta \psi$ by the parameter $(\beta, \eta, p, w)$ representing congruent classes of Kirchhoff elastic rods (Proposition 7.1). We note that the functions $\Delta \theta(\beta, \eta, p, w)$, $\Delta \psi(\beta, \eta, p, w)$ are explicitly expressed in terms of the complete elliptic integral of the first and third kinds. We give an equivalent condition for $\{\gamma, M\}$ to be a closed Kirchhoff elastic rod, that is, a Kirchhoff elastic rod whose centerline is a periodic curve.

THEOREM 1.2 (cf. Theorem 7.2). Let $\{\gamma, M\}$ be a Kirchhoff elastic rod in $S^{3}$ corresponding to $(\beta, \eta, p, w)$. Assume that $\{\gamma, M\}$ is neither of the helix case nor of the Hasimoto soliton case. Then, $\{\gamma, M\}$ is a closed Kirchhoff elastic rod if and only if $\Delta \theta(\beta, \eta, p, w) /(2 \pi)$ and $\Delta \psi(\beta, \eta, p, w) /(2 \pi)$ are both rational numbers.

In Section 8, by making use of this theorem, we give an example of closed Kirchhoff elastic rods. Specifically, we construct a smooth two-parameter family of closed Kirchhoff elastic rods including both helices and non-helices.

THEOREM 1.3 (cf. Theorem 8.1). There exists a smooth two-parameter family $\left\{\gamma^{\lambda, \omega}, M^{\lambda, \omega}\right\}(0 \leqslant \lambda \ll 1,|\omega| \ll 1)$ of closed Kirchhoff elastic rods satisfying the following. If $\lambda=0$, then $\gamma^{\lambda, \omega}$ is a helix, and if $\lambda \neq 0$, then $\gamma^{\lambda, \omega}$ is not a helix. Moreover, if $\lambda_{1}$, $\lambda_{2} \neq 0$ and $\left(\lambda_{1}, \omega_{1}\right) \neq\left(\lambda_{2}, \omega_{2}\right)$ hold, then $\gamma^{\lambda_{1}, \omega_{1}}$ and $\gamma^{\lambda_{2}, \omega_{2}}$ are not congruent.

A closed Kirchhoff elastic rod whose centerline is a helix seems to be a relatively trivial object. By the above theorem, we also see that there exist infinitely many non-trivial closed Kirchhoff elastic rods.

2. The Euler-Lagrange equation. In this section, we calculate the first variation formula for the energy $\mathfrak{T}$ and derive the Euler-Lagrange equation. Unless otherwise specified, 
all manifolds, curves, vector fields, etc., are assumed to be smooth $\left(=C^{\infty}\right)$. Let $\mathcal{M}$ be a Riemannian manifold of dimension $n(\geqslant 2)$. We denote by $\langle$,$\rangle the Riemannian metric of$ $\mathcal{M}$. Let $\gamma=\gamma(t):[0, l] \rightarrow \mathcal{M}$ be a regular curve. Let $\gamma^{\prime}$ be the tangent vector to $\gamma$, $v(t)=\left|\gamma^{\prime}(t)\right|=\left\langle\gamma^{\prime}(t),\left.\gamma^{\prime}(t)\right|^{1 / 2}\right.$ the speed, and $T(t)=(1 / v(t)) \gamma^{\prime}(t)$ the unit tangent vector. We denote the length of $\gamma$ by $\mathfrak{L}(\gamma)=\int_{0}^{l} v d t$. We denote by $T \mathcal{M}$ the tangent bundle of $\mathcal{M}$, and by $\nabla$ the Levi-Civita connection in $T \mathcal{M}$, and use the symbols $\nabla_{t}=\nabla_{\partial / \partial t}=\nabla_{\gamma^{\prime}}$. Also, we denote by $T^{\perp} \mathcal{M}$ the normal bundle along $\gamma$, and by $\nabla^{\perp}$ the normal connection in $T^{\perp} \mathcal{M}$.

To describe how the elastic rod is twisted, we utilize an orthonormal frame field $M=$ $\left(M_{1}, M_{2}, \ldots, M_{n-1}\right)$ in $T^{\perp} \mathcal{M}$. We consider the pair $\{\gamma, M\}$ of $\gamma$ and $M$. In this paper, we call such a pair $\{\gamma, M\}$ a curve with adapted orthonormal frame, and $\gamma$ the centerline of $\{\gamma, M\}$. Note that $\left(T(t), M_{1}(t), \ldots, M_{n-1}(t)\right)$ is an orthonormal basis of $T_{\gamma(t)} \mathcal{M}$ for each $t$.

Now, let $v$ be a positive constant. We define the energy $\mathfrak{T}$ as follows:

$$
\mathfrak{T}(\{\gamma, M\})=\int_{0}^{l}\left|\nabla_{T} T\right|^{2} v d t+v \sum_{i=1}^{n-1} \int_{0}^{l}\left|\nabla_{T}^{\perp} M_{i}\right|^{2} v d t .
$$

Note that $\mathfrak{T}$ is invariant under reparametrization of $t$.

Let $P, Q \in \mathcal{M}$. Let $X=\left(X_{0}, X_{1}, \ldots, X_{n-1}\right)$ be an orthonormal frame of $T_{P} \mathcal{M}$, and $Y=\left(Y_{0}, Y_{1}, \ldots, Y_{n-1}\right)$ an orthonormal frame of $T_{Q} \mathcal{M}$. We denote by $\mathcal{B}(0, P, X ; l, Q, Y)$, or simply by $\mathcal{B}$, the space of $\{\gamma, M\}$ satisfying the following conditions:

$$
\begin{array}{lll}
\gamma(0)=P, & T(0)=X_{0}, & M(0)=\left(X_{1}, \ldots, X_{n-1}\right), \\
\gamma(l)=Q, & T(l)=Y_{0}, & M(l)=\left(Y_{1}, \ldots, Y_{n-1}\right) .
\end{array}
$$

We denote by $\mathcal{U B}(0, P, X ; l, Q, Y)$, or simply by $\mathcal{U B}$, the space of all elements $\{\gamma, M\}$ of $\mathcal{B}(0, P, X ; l, Q, Y)$ satisfying $v(t) \equiv 1$, and by $\mathcal{B}_{l}(0, P, X ; l, Q, Y)$, or simply by $\mathcal{B}_{l}$, the space of all elements $\{\gamma, M\}$ of $\mathcal{B}(0, P, X ; l, Q, Y)$ satisfying $\mathfrak{L}(\gamma)=l$. Note that $\mathcal{U B} \subset$ $\mathcal{B}_{l} \subset \mathcal{B}$.

Now we consider a variation of a curve with adapted orthonormal frame. Let $\{\gamma, M\}$ be a curve with adapted orthonormal frame defined on $[0, l]$, and $\lambda_{0}$ a positive number. Consider a map $\tilde{\gamma}:\left(-\lambda_{0}, \lambda_{0}\right) \times[0, l] \rightarrow \mathcal{M}$, and $\tilde{M}=\left(\tilde{M}_{1}, \ldots, \tilde{M}_{n-1}\right)$ an ordered set of $n-1$ vector fields along $\tilde{\gamma}$. We denote by $\gamma^{\lambda}$ the curve defined by $\gamma^{\lambda}(t)=\tilde{\gamma}(\lambda, t)$, and by $M^{\lambda}$ the ordered pair of $n-1$ vector fields along $\gamma^{\lambda}$ defined by $M^{\lambda}(t)=\tilde{M}(\lambda, t)=$ $\left(\tilde{M}_{1}(\lambda, t), \ldots, \tilde{M}_{n-1}(\lambda, t)\right)$. The pair $\{\tilde{\gamma}, \tilde{M}\}$, or the family $\left\{\left\{\gamma^{\lambda}, M^{\lambda}\right\}\right\}\left(|\lambda|<\lambda_{0}\right)$, is called a variation of $\{\gamma, M\}$ if $\left\{\gamma^{\lambda}, M^{\lambda}\right\}$ is a curve with adapted orthonormal frame for each $\lambda \in$ $\left(-\lambda_{0}, \lambda_{0}\right)$ and $\left\{\gamma^{0}, M^{0}\right\}=\{\gamma, M\}$.

We denote $\mathcal{B}, \mathcal{B}_{l}$, or $\mathcal{U B}$ by $\mathcal{C}$. Let $\{\gamma, M\} \in \mathcal{C}$. A variation $\left\{\gamma^{\lambda}, M^{\lambda}\right\}\left(|\lambda|<\lambda_{0}\right)$ of $\{\gamma, M\}$ is called a $\mathcal{C}$-variation if $\left\{\gamma^{\lambda}, M^{\lambda}\right\} \in \mathcal{C}$ for each $\lambda \in\left(-\lambda_{0}, \lambda_{0}\right)$. Also, $\{\gamma, M\}$ is called a $\mathcal{C}$-critical point of $\mathfrak{T}$ if

$$
\left.\frac{d}{d \lambda}\right|_{\lambda=0} \mathfrak{T}\left(\left\{\gamma^{\lambda}, M^{\lambda}\right\}\right)=0
$$

for any $\mathcal{C}$-variation $\left\{\gamma^{\lambda}, M^{\lambda}\right\}$ of $\{\gamma, M\}$. We consider the following variational problem:

Problem. Determine the $\mathcal{U} \mathcal{B}$-critical points of the energy $\mathfrak{T}$. 
Here note that our configuration space is $\mathcal{U B}$, because the elastic rod is assumed to be inextensible. We do not treat directly the above boundary value problem, but derive the EulerLagrange equation in the rest of this section. In the following sections, we solve it in the case where $\mathcal{M}=S^{3}$.

We now introduce the "tangent space" to $\mathcal{B}$. Let $\{\gamma, M\} \in \mathcal{B}$, and $\left\{\gamma^{\lambda}, M^{\lambda}\right\}\left(|\lambda|<\lambda_{0}\right)$ a variation of $\{\gamma, M\}$. We denote the tangent vector, the speed, the unit tangent, and the curvature of $\gamma^{\lambda}$ by $V^{\lambda}(t)=\tilde{V}(\lambda, t), v^{\lambda}(t)=\tilde{v}(\lambda, t), T^{\lambda}(t)=\tilde{T}(\lambda, t)$, and $k^{\lambda}(t)=\tilde{k}(\lambda, t)$, respectively. Then, $\tilde{k}(\lambda, t)=\left|\left(\nabla_{\tilde{T}} \tilde{T}\right)(\lambda, t)\right|$, where $\nabla_{\tilde{T}}=\nabla_{(1 / \tilde{v})(\partial / \partial t)}^{\tilde{\gamma}^{-1} T \mathcal{M}}$. We define $\tilde{\Lambda}$ and $\tilde{f}_{i}^{j}$ as follows:

$$
\begin{aligned}
\tilde{\Lambda}(\lambda, t) & =\frac{\partial \tilde{\gamma}}{\partial \lambda}(\lambda, t), \\
\tilde{f}_{i}^{j}(\lambda, t) & =\left\langle\nabla_{\tilde{\Lambda}} \tilde{M}_{i}, \tilde{M}_{j}\right\rangle, \quad 1 \leqslant i \leqslant n-1,
\end{aligned}
$$

where $\nabla_{\tilde{\Lambda}}=\nabla_{\partial / \partial \lambda}^{\tilde{\gamma}^{-1} T \mathcal{M}}$. Let $\tilde{F}(\lambda, t)$ be the $(n-1) \times(n-1)$ matrix with $j i$ components $\tilde{f}_{i}^{j}(\lambda, t)$. We denote the restriction of $\tilde{\Lambda}, \tilde{f}_{i}^{j}$ and $\tilde{F}$ to $\lambda=0$ by $\Lambda(t), f_{i}^{j}(t)$ and $F(t)$, respectively, and denote $\left.\nabla_{\tilde{\Lambda}}\right|_{\lambda=0}$ by $\nabla_{\Lambda}$. In the same way as in page 99 of [9], it is verified that

$$
\left\langle\nabla_{\Lambda} \tilde{M}_{i}, T\right\rangle=-\left\langle M_{i}, \nabla_{T} \Lambda\right\rangle .
$$

Thus, the tangential component of $\nabla_{\Lambda} \tilde{M}_{i}$ is determined by $\Lambda$. Therefore, there is a one-to-one correspondence between the pair $\left(\Lambda, \nabla_{\Lambda} \tilde{M}_{1}, \ldots, \nabla_{\Lambda} \tilde{M}_{n-1}\right)$ and the pair $(\Lambda, F)$. We call the pair $(\Lambda, F)$ the variation vector field of the variation $\left\{\gamma^{\lambda}, M^{\lambda}\right\}$.

We define the following vector space:

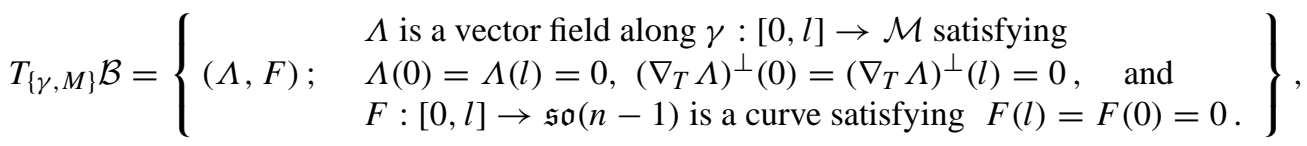

where $\left(\nabla_{T} \Lambda\right)^{\perp}$ is the normal component of $\nabla_{T} \Lambda$, so that $\left(\nabla_{T} \Lambda\right)^{\perp}=\nabla_{T} \Lambda-\left\langle\nabla_{T} \Lambda, T\right\rangle T$, and $\mathfrak{s o}(n-1)$ stands for the vector space of all $(n-1) \times(n-1)$ skew symmetric matrices. Then the following lemma holds. Therefore, the vector space $T_{\{\gamma, M\}} \mathcal{B}$ can be viewed as the tangent space to $\mathcal{B}$ at $\{\gamma, M\}$. (The proof is similar to those of Lemma 2.8 of [9] and Lemma 3.1 of $[10]$.)

Lemma 2.1. Let $\{\gamma, M\} \in \mathcal{B}$. If $\left\{\gamma^{\lambda}, M^{\lambda}\right\}(|\lambda| \ll 1)$ is a $\mathcal{B}$-variation of $\{\gamma, M\}$, then the variation vector field $(\Lambda, F)$ belongs to $T_{\{\gamma, M\}} \mathcal{B}$. Conversely, for an arbitrary $(\Lambda, F) \in$ $T_{\{\gamma, M\}} \mathcal{B}$, there exists a $\mathcal{B}$-variation $\left\{\gamma^{\lambda}, M^{\lambda}\right\}$ whose variation vector field is $(\Lambda, F)$.

In order to apply the Lagrange multiplier principle, we define a functional $\mathfrak{T}^{\mu}$ by

$$
\mathfrak{T}^{\mu}(\{\gamma, M\})=\mathfrak{T}(\{\gamma, M\})+\mu \mathfrak{L}(\gamma)
$$

for a constant $\mu \in \boldsymbol{R}$. The first variation formula for $\mathfrak{T}^{\mu}$ is calculated as follows (cf. [9], [10], [17]). We use the sign convention of the curvature tensor $R$ corresponding to that of [12], that is, $R(X, Y) Z=\nabla_{X} \nabla_{Y} Z-\nabla_{Y} \nabla_{X} Z-\nabla_{[X, Y]} Z$. 
Proposition 2.2. Let $\{\gamma, M\} \in \mathcal{B}$, and $\left\{\gamma^{\lambda}, M^{\lambda}\right\}(|\lambda| \ll 1)$ a $\mathcal{B}$-variation of $\{\gamma, M\}$. Let $(\Lambda, F)$ denote the variation vector field of the variation $\left\{\gamma^{\lambda}, M^{\lambda}\right\}$. Then,

$$
\begin{aligned}
\left.\frac{d}{d \lambda}\right|_{\lambda=0} \mathfrak{T}^{\mu}\left(\left\{\gamma^{\lambda}, M^{\lambda}\right\}\right)=\int_{0}^{l}\left\langle\nabla _ { T } \left[ 2\left(\nabla_{T}\right)^{2} T+\left(3\left|\nabla_{T} T\right|^{2}-\mu+v \sum_{i=1}^{n-1}\left|\nabla_{T}^{\perp} M_{i}\right|^{2}\right) T\right.\right. \\
\left.\quad-4 v \sum_{i=1}^{n-1}\left\langle\nabla_{T} T, M_{i}\right\rangle \nabla_{T}^{\perp} M_{i}\right] \\
\left.+2 R\left(\nabla_{T} T, T\right) T+2 v \sum_{i=1}^{n-1} R\left(\nabla_{T}^{\perp} M_{i}, M_{i}\right) T, \Lambda\right\rangle v d t \\
-2 v \sum_{i, j=1}^{n-1} \int_{0}^{l}\left(T\left\langle\nabla_{T}^{\perp} M_{i}, M_{j}\right\rangle\right) f_{i}^{j} v d t .
\end{aligned}
$$

Since the functional $\mathfrak{T}$ is invariant under reparametrization of $t$, we see that $\{\gamma, M\}$ is a $\mathcal{U B}$-critical point of $\mathfrak{T}$ if and only if $\{\gamma, M\}$ is a $\mathcal{B}_{l}$-critical point of $\mathfrak{T}$. Thus, by using the Lagrange multiplier principle, we have the following lemma.

Lemma 2.3. Let $\{\gamma, M\} \in \mathcal{U B}$. Suppose that $\gamma$ is not a geodesic. Then $\{\gamma, M\}$ is a $\mathcal{U B}$-critical point of $\mathfrak{T}$ if and only if there exists a constant $\mu \in \boldsymbol{R}$ such that $\{\gamma, M\}$ is a $\mathcal{B}$-critical point of $\mathfrak{T}^{\mu}$

Hence, by Proposition 2.2 and Lemma 2.3, we obtain

Proposition 2.4. Let $\{\gamma, M\} \in \mathcal{U B}$. Suppose that $\gamma$ is not a geodesic. Then $\{\gamma, M\}$ is a $\mathcal{U B}$-critical point of $\mathfrak{T}$ if and only if $\{\gamma, M\}$ satisfies the following equations for some real constant $\mu$.

$$
\begin{aligned}
& \nabla_{T}\left[2\left(\nabla_{T}\right)^{2} T+\left(3\left|\nabla_{T} T\right|^{2}-\mu+v \sum_{i=1}^{n-1}\left|\nabla_{T}^{\perp} M_{i}\right|^{2}\right) T-4 v \sum_{i=1}^{n-1}\left\langle\nabla_{T} T, M_{i}\right\rangle \nabla_{T}^{\perp} M_{i}\right] \\
& \quad+2 R\left(\nabla_{T} T, T\right) T+2 v \sum_{i=1}^{n-1} R\left(\nabla_{T}^{\perp} M_{i}, M_{i}\right) T=0
\end{aligned}
$$

$$
\frac{d}{d t}\left\langle\nabla_{T}^{\perp} M_{i}, M_{j}\right\rangle=0, \quad 1 \leqslant i, j \leqslant n-1 .
$$

Also, the constant $\mu$ is uniquely determined.

In this paper, we define a Kirchhoff elastic rod as follows.

DEFINITION 2.5. Let $\{\gamma, M\}$ be a unit-speed curve with adapted orthonormal frame. Then $\{\gamma, M\}$ is said to be a Kirchhoff elastic rod if $\{\gamma, M\}$ satisfies (2.1) and (2.2) for some $\mu \in \boldsymbol{R}$.

From now on, let $\mathcal{M}$ be $\boldsymbol{R}^{3}, S^{3}$ or $H^{3}$ of constant sectional curvature $G$. We fix an orientation of $\mathcal{M}$, and denote by $\times$ the vector product. Then (2.2) is equivalent to that there 
exists a constant $a \in \boldsymbol{R}$ such that

$$
\left\langle\nabla_{T}^{\perp} M_{1}, T \times M_{1}\right\rangle=a .
$$

Substituting (2.3) to (2.1) yields

$$
\nabla_{T}\left[2\left(\nabla_{T}\right)^{2} T+\left(3\left|\nabla_{T} T\right|^{2}-(\mu-2 G)+2 v a^{2}\right) T-4 v a T \times \nabla_{T} T\right]=0 .
$$

Therefore, $\{\gamma, M\}$ is a Kirchhoff elastic rod if and only if there exist two constants $a, \mu$ such that (2.4) and (2.3) hold. In this paper, we call the constant $a$ the twist rate of $\{\gamma, M\}$. (In [9], the term "torsional parameter" is used.) When $\gamma$ is not a geodesic, the constant $\mu$ is uniquely determined, and is called the Lagrange multiplier of $\{\gamma, M\}$.

We denote by $\mathcal{R}_{t}(\varphi)$ the rotation on the normal vector space $T_{\gamma(t)}^{\perp} \mathcal{M}$ by angle $\varphi$. (Its direction is determined by the requirement that $\mathcal{R}_{t}(\pi / 2)\left(Y_{1}\right)=Y_{2}$, where $\left(T, Y_{1}, Y_{2}\right)$ is a positive orthonormal frame of $T_{\gamma(t)} \mathcal{M}$.) For a function $\psi(t)$ and a normal vector field $X(t)$ along $\gamma, \mathcal{R}(\psi) X$ is defined by $(\mathcal{R}(\psi) X)(t)=\mathcal{R}_{t}(\psi(t)) X(t)$.

Let $\{\gamma, M\}$ be a curve with adapted orthonormal frame, and $W_{1}$ a unit normal along $\gamma$ satisfying $\nabla_{T}^{\perp} W_{1}=0$. Suppose that $M_{1}$ is expressed as $M_{1}=\mathcal{R}(\psi) W_{1}$. Then, in the same way as in Proposition 2.2 of [9], we have $T \psi=\left\langle\nabla_{T}^{\perp} M_{1}, T \times M_{1}\right\rangle$. Therefore, if $\{\gamma, M\}$ is a Kirchhoff elastic rod with twist rate $a$, then $M_{1}$ is expressed as $M_{1}=\mathcal{R}\left(a t+\psi_{0}\right) W_{1}$, where $\psi_{0} \in \boldsymbol{R}$. The physical meaning of (2.3) is that the twist of a Kirchhoff elastic rod is uniformly distributed along the curve.

The equation (2.4) with $v=0$ is just the equation of elastica (cf. (1.1) of [17]). It is easily verified that $\{\gamma, M\}$ is a Kirchhoff elastic rod with twist rate 0 if and only if $\gamma$ is an elastica and $M$ is parallel with respect to the normal connection along $\gamma$.

Finally, we note that if $\{\gamma(t), M(t)\}$ is a Kirchhoff elastic rod in $\mathcal{M}=\boldsymbol{R}^{3}, S^{3}$ or $H^{3}$, then both $\gamma(t)$ and $M(t)$ are real analytic in $t$. The proof is similar to that of Proposition 2.13 in [9].

3. Curvature and torsion of Kirchhoff elastic rods. In this section, we obtain explicit expressions of the curvature and torsion of the centerline of a Kirchhoff elastic rod in $\mathcal{M}=\boldsymbol{R}^{3}, S^{3}$ or $H^{3}$ of constant sectional curvature $G$, and introduce a parametrization of the space of congruent classes of Kirchhoff elastic rods (Proposition 3.1). In the case of $S^{3}$, we introduce, in the last of this section, another parametrization of the space, which is mainly used in Sections 6, 7 and 8. From now on, we always assume that the centerline of a curve with adapted orthonormal frame is a unit-speed curve.

We define a congruent class of curves with adapted orthonormal frames. Consider the following transformations which transform $\{\gamma(t), M(t)\}$ into

$\left\{\gamma\left( \pm\left(t-t_{1}\right)\right), M\left( \pm\left(t-t_{1}\right)\right)\right\}$, where $t_{1} \in \boldsymbol{R}$,

(3.2) $\left\{S \circ \gamma, S_{*} M\right\}$, where $S$ is an isometry of $\mathcal{M}$, and $S_{*}$ is the differential map of $S$,

(3.3) $\{\gamma, M \varphi\}$, where $\varphi \in O(2)$, 
respectively. A map of finite compositions of the above transformations (3.1), (3.2), and (3.3) is called a congruent transformation. Two curves with adapted orthonormal frames $\{\gamma, M\}$ and $\{\tilde{\gamma}, \tilde{M}\}$ are called congruent if $\{\gamma, M\}$ can be transformed into $\{\tilde{\gamma}, \tilde{M}\}$ by a congruent transformation.

First, we examine a Kirchhoff elastic rod whose centerline is a geodesic. Let $\{\gamma, M\}$ be a curve with adapted orthonormal frame such that $\gamma$ is a geodesic, and let $W_{1}(t)$ be a unit normal along $\gamma$ satisfying $\nabla_{T}^{\perp} W_{1}=0$. Then (2.4) holds for any $a, \mu$. Thus, $\{\gamma, M\}$ is a Kirchhoff elastic rod if and only if $M_{1}$ is expressed as $M_{1}(t)=\mathcal{R}\left(a t+\psi_{0}\right) W_{1}(t)$, where $a$ and $\psi_{0}$ are arbitrary two constants. A Kirchhoff elastic rod whose centerline is a geodesic is a relatively trivial object.

In the rest of the paper, we always assume that the centerline of a Kirchhoff elastic rod is not a geodesic, that is, the curvature of $\gamma$ is not identically zero.

Let $k$ denote the curvature of $\gamma$. Suppose that $k$ is positive everywhere. We denote by $\tau$ the torsion, and by $(T, N, B)$ the Frenet frame of $\gamma$, where the direction of $B$ is determined by the requirement $B=T \times N$. Then the following Frenet formulas hold.

$$
\nabla_{T} T=k N, \quad \nabla_{T} N=-k T+\tau B, \quad \nabla_{T} B=-\tau N .
$$

Suppose that $M_{1}(t)$ is expressed as $M_{1}(t)=\mathcal{R}(\varphi(t)) N(t)$, where $\varphi(t)$ is a function of $t$. Then, by a calculation similar to that in page 105 of [9], we have $\left\langle\nabla_{T}^{\perp} M_{1}, T \times M_{1}\right\rangle=\varphi^{\prime}+\tau$. Thus, if $\{\gamma, M\}$ is a Kirchhoff elastic rod, then

$$
\varphi^{\prime}+\tau=a .
$$

In the proposition below, we derive the equations of $k$ and $\tau$ by substituting (3.4) to (2.4), and solve them in terms of Jacobi sn function (cf. Lemma 4.1 of [9], Section 2 of [17]). Let sn, cn, dn denote Jacobi sn, cn, dn functions, respectively (cf. [3]).

PROPOSITION 3.1. The space of all congruent classes of Kirchhoff elastic rods (except geodesics) in $\mathcal{M}=\boldsymbol{R}^{3}, S^{3}$ or $H^{3}$ defined on $\boldsymbol{R}$ corresponds to the parameter space $\mathcal{D}=$ $\tilde{\mathcal{D}} / \sim$, where

$$
\tilde{\mathcal{D}}=\left\{\left(a_{+}, \alpha_{1}, \alpha_{2}, \alpha_{3}\right) \in \boldsymbol{R}^{4} ;-\alpha_{1} \leqslant 0 \leqslant \alpha_{2} \leqslant \alpha_{3}, \alpha_{3}>0\right\},
$$

and the equivalence relation $\sim$ is defined as follows: If $\alpha_{1}=0$ or $\alpha_{2}=0$, then $\left(a_{+}, \alpha_{1}, \alpha_{2}, \alpha_{3}\right) \sim\left(-a_{+}, \alpha_{1}, \alpha_{2}, \alpha_{3}\right)$.

An element $\left[\left(a_{+}, \alpha_{1}, \alpha_{2}, \alpha_{3}\right)\right]$ of $\mathcal{D}$ corresponds to the congruent class of Kirchhoff elastic rods with twist rate $\pm a_{+}$, whose squared curvature $u(t)$ and torsion $\tau(t)$ are expressed as follows:

$$
\begin{gathered}
u(t)=\alpha_{3}\left(1-q^{2} \operatorname{sn}^{2}\left(y\left(t-t_{0}\right), p\right)\right), \\
\tau(t)= \pm\left(\frac{\sqrt{\alpha_{1} \alpha_{2} \alpha_{3}}}{2 u(t)}+v a_{+}\right),
\end{gathered}
$$


where $t_{0} \in \boldsymbol{R}$ is a constant and

$$
p=\sqrt{\frac{\alpha_{3}-\alpha_{2}}{\alpha_{3}+\alpha_{1}}}, \quad q=\sqrt{\frac{\alpha_{3}-\alpha_{2}}{\alpha_{3}}}, \quad y=\frac{\sqrt{\alpha_{1}+\alpha_{3}}}{2} .
$$

Here the double sign of $a_{+}$and that of the right hand side of (3.7) are in the same order.

Proof. Let $\{\gamma(t), M(t)\}$ be a Kirchhoff elastic rod defined on $\boldsymbol{R}$. Let $a$ and $\mu$ denote the twist rate and Lagrange multiplier of $\{\gamma, M\}$, respectively. We determine $\left(a_{+}, \alpha_{1}, \alpha_{2}, \alpha_{3}\right) \in$ $\tilde{\mathcal{D}}$ by dividing the argument into three cases.

Case 1. Generic case: Nonvanishing and nonconstant curvature case.

We first consider the case that the curvature $k$ of $\gamma$ is not constant and $k(t)>0$ for all $t$. Substituting the Frenet formulas (3.4) to the Euler-Lagrange equation (2.4), we have

$$
2 k^{\prime \prime}+k^{3}+\left(2 v a^{2}-(\mu-2 G)\right) k-2 k \tau(\tau-2 v a)=0,
$$

$$
k^{2}(\tau-v a)=b,
$$

where $b$ is a constant.

Using the substitution $\tau=b / k^{2}+v a$ and multiplication by $k^{\prime}$ and integration, we obtain

$$
\left(k^{\prime}\right)^{2}+\frac{k^{4}}{4}+\frac{1}{2}\left(2 v a^{2}-(\mu-2 G)+2 v^{2} a^{2}\right) k^{2}+\frac{b^{2}}{k^{2}}=c,
$$

where $c$ is a constant. For $\{\gamma, M\}$, the four constants $\mu, a, b, c$ are determined.

By the change of variable $u=k^{2}$, we get

$$
\left(u^{\prime}\right)^{2}=-u^{3}-2\left(2 v a^{2}-(\mu-2 G)+2 v^{2} a^{2}\right) u^{2}+4 c u-4 b^{2} .
$$

Denote by $Q(u)$ the right hand side of (3.12). Since $Q(0)=-4 b^{2} \leqslant 0$, the minimum real root of the cubic equation $Q(u)=0$ is nonpositive, which we denote by $-\alpha_{1}$. Furthermore, since $u^{\prime}$ is not identically zero, there exists some $u>0$ such that the cubic polynomial $Q(u)$ is positive. Therefore the equation $Q(u)=0$ has at least one positive root. We denote by $\alpha_{3}$ the maximum of these positive roots, and by $\alpha_{2}$ the other root of the cubic equation. Since $Q(0) \leqslant 0$, we have $\alpha_{2} \geqslant 0$. Also, since there exists some $u>0$ such that $Q(u)>0$, we see $\alpha_{2} \neq \alpha_{3}$. Hence, $-\alpha_{1} \leqslant 0 \leqslant \alpha_{2}<\alpha_{3}$. The real numbers $\alpha_{1}, \alpha_{2}, \alpha_{3}$ are related to the parameters $\mu, a, b, c$ by

$$
\begin{gathered}
2\left(2 v a^{2}-(\mu-2 G)+2 v^{2} a^{2}\right)=\alpha_{1}-\alpha_{2}-\alpha_{3}, \\
-4 c=-\alpha_{1} \alpha_{2}+\alpha_{2} \alpha_{3}-\alpha_{1} \alpha_{3}, \\
4 b^{2}=\alpha_{1} \alpha_{2} \alpha_{3} .
\end{gathered}
$$

The solution of the ordinary differential equation $\left(u^{\prime}\right)^{2}=Q(u)$ is expressed in terms of Jacobi sn function and the parameters $\alpha_{1}, \alpha_{2}, \alpha_{3}$ as (3.6), where $t_{0} \in \boldsymbol{R}$ and $p, q, y$ are defined by (3.8).

Since $k(t)$ is assumed to be positive everywhere, we have

$$
\alpha_{2} \neq 0 \quad \text { or } \quad \alpha_{1}=\alpha_{2}=0 .
$$


Let $K(p)$ denote the complete elliptic integral of the first kind (cf. [3], [17]). If $\alpha_{2} \neq 0$, then $u(t)$ is a $(2 K(p) / y)$-periodic function, that is, a periodic function with primitive period $2 K(p) / y$, and attains the maximum (resp. minimum) value $\alpha_{3}$ (resp. $\left.\alpha_{2}\right)$ at $t_{0}+2 m K(p) / y$ (resp. $\left.t_{0}+(2 m+1) K(p) / y\right)$, where $m$ is an integer. If $\alpha_{1}=\alpha_{2}=0(\Leftrightarrow p=1)$, then $u(t)$ becomes $\alpha_{3} \operatorname{sech}^{2}\left(y\left(t-t_{0}\right)\right)$, which is not periodic and attains the maximum value $\alpha_{3}$ at $t_{0}$, and converges to 0 as $t \rightarrow \pm \infty$.

We set $a_{+}=a$ if $b \geqslant 0$, and $a_{+}=-a$ if $b<0$. Then, by (3.10) and (3.15), we have (3.7), where the upper sign is taken if $b \geqslant 0$, and the lower sign if $b<0$.

For $\{\gamma, M\},\left(a_{+}, \alpha_{1}, \alpha_{2}, \alpha_{3}\right) \in \tilde{\mathcal{D}}$ satisfying $\alpha_{2} \neq \alpha_{3}$ and (3.16) is determined as above. The squared curvature and the torsion of $\gamma$ are expressed as (3.6) and (3.7), and the twist rate is $\pm a_{+}$.

Case 2. Constant curvature case.

We consider the case that $k(t)$ is a positive constant. The constants $\mu, a, b, c$ are determined in the same way as in Case 1. By (3.10), the torsion $\tau(t)$ is also constant. Therefore, $\gamma$ is a helix. We formally define the parameters $\alpha_{1}, \alpha_{2}, \alpha_{3}$ in terms of the squared curvature $u$, the torsion $\tau$, and the twist rate $a$ as follows.

$$
\alpha_{1}=4(\tau-v a)^{2}, \quad \alpha_{2}=\alpha_{3}=u \text {. }
$$

We define $a_{+}$in the same way as in Case 1.

For $\{\gamma, M\},\left(a_{+}, \alpha_{1}, \alpha_{2}, \alpha_{3}\right) \in \tilde{\mathcal{D}}$ satisfying $\alpha_{2}=\alpha_{3}$ is determined as above, and the squared curvature, the torsion, and the twist rate are expressed as (3.6), (3.7), and $\pm a_{+}$, respectively, where the upper sign is taken if $b \geqslant 0$, and the lower sign if $b<0$. Here we note that it follows from (3.9) and (3.11) that

$$
\mu=u+2 v a^{2}-2 \tau(\tau-2 v a)+2 G, \quad c=-\frac{u^{2}}{4}+2 u(\tau-v a)^{2} .
$$

Therefore, $\alpha_{1}, \alpha_{2}, \alpha_{3}$ defined as above satisfy the relations (3.13), (3.14) and (3.15).

Case 3. The case that $\gamma$ has inflection points.

We finally consider the case that $\gamma$ has an inflection point, that is, a point where $k(t)$ vanishes. In this case, we restrict the argument of Case 1 to an open interval $I$ on which $k(t)$ is positive. Then there exist $\alpha_{1}, \alpha_{2}, \alpha_{3}$ satisfying $-\alpha_{1} \leqslant 0 \leqslant \alpha_{2}<\alpha_{3}$, and the squared curvature $u$ is expressed as (3.6) on $I$. This expression is valid for the whole $\boldsymbol{R}$, because $u$ is real analytic on $\boldsymbol{R}$. Thus we can verify that the parameters $\alpha_{1}, \alpha_{2}, \alpha_{3}$ determined as above are independent of the choice of the open interval $I$. Also, since there exists a point such that $u(t)=0$, we see $q=1$ and $p \neq 1$. Thus, $\alpha_{2}=0$ and $\alpha_{1} \neq 0$. Consequently, the curvature is expressed as

$$
k(t)=\sqrt{\alpha_{3}}\left|\operatorname{cn}\left(y\left(t-t_{0}\right), p\right)\right|,
$$

which vanishes at $t=t_{0}+(2 m+1) K(p) / y$, and the torsion $\tau$ is $v a$ except at the inflection points. We define $a_{+}$in the same way as in the other cases. Since $b=0, a_{+}=a$. 
For $\{\gamma, M\},\left(a_{+}, \alpha_{1}, \alpha_{2}, \alpha_{3}\right) \in \tilde{\mathcal{D}}$ satisfying $\alpha_{2}=0$ and $\alpha_{1} \neq 0$ is determined as above, and the squared curvature, the torsion, and the twist rate are expressed as (3.6), (3.7), and $a_{+}$, respectively, where the upper sign is taken.

In each case, $\{\gamma, M\}$ determines $\left(a_{+}, \alpha_{1}, \alpha_{2}, \alpha_{3}\right) \in \tilde{\mathcal{D}}$ as mentioned above. We can check that the parameters $\mu, a, b, c, \alpha_{1}, \alpha_{2}$ and $\alpha_{3}$ are invariant under the transformations (3.1) and (3.3). Also, if $S$ is an orientation-preserving isometry, then they are invariant under (3.2), and if $S$ is an orientation-reversing isometry, then they are transformed to $\mu,-a,-b, c, \alpha_{1}$, $\alpha_{2}$ and $\alpha_{3}$ by (3.2). Therefore, if $b \neq 0$, then $\left(a_{+}, \alpha_{1}, \alpha_{2}, \alpha_{3}\right)$ is invariant under congruent transformations. Also, if $b=0$, that is, $\alpha_{1}=0$ or $\alpha_{2}=0$, then it is invariant or transformed to $\left(-a_{+}, \alpha_{1}, \alpha_{2}, \alpha_{3}\right)$. Consequently, $\left[\left(a_{+}, \alpha_{1}, \alpha_{2}, \alpha_{3}\right)\right] \in \mathcal{D}$ is determined only by a congruent class of $\{\gamma, M\}$.

Conversely, we take an arbitrary $\left[\left(a_{+}, \alpha_{1}, \alpha_{2}, \alpha_{3}\right)\right] \in \mathcal{D}$. In the same way as in the argument in pages 122-123 of [9], we can construct a unique Kirchhoff elastic $\operatorname{rod}\{\gamma, M\}$ (up to congruent transformations) which yields $\left[\left(a_{+}, \alpha_{1}, \alpha_{2}, \alpha_{3}\right)\right]$. Therefore the above map which sends a congruent class of Kirchhoff elastic rods to $\left[\left(a_{+}, \alpha_{1}, \alpha_{2}, \alpha_{3}\right)\right](\in \mathcal{D})$ is bijective.

For the use in Lemma 6.3, we define the generalized Frenet frame along $\gamma$ in the case where $\gamma$ has inflection points. In this case, the Frenet frame $(T, N, B)$ is not defined at inflection points. However, it is verified that there exists a real analytic orthonormal frame field $(T, \hat{N}, \hat{B})$ along $\gamma$ such that

$$
\nabla_{T} T=\hat{k} \hat{N}, \quad \nabla_{T} \hat{N}=-\hat{k} T+\tau \hat{B}, \quad \nabla_{T} \hat{B}=-\tau \hat{N},
$$

where

$$
\hat{k}(t)=\sqrt{\alpha_{3}} \operatorname{cn}\left(y\left(t-t_{0}\right), p\right), \quad \tau(t)=v a .
$$

We call $\hat{k}$ the signed curvature of $\gamma$, and $(T, \hat{N}, \hat{B})$ the generalized Frenet frame along $\gamma$. Then, at a point where $\hat{k}(t)>0$ (resp. $\hat{k}(t)<0)$, the curvature is equal to $\hat{k}(t)($ resp. $-\hat{k}(t))$, and the (ordinary) Frenet frame $(T, N, B)$ is equal to $(T, \hat{N}, \hat{B})($ resp. $(T,-\hat{N},-\hat{B}))$. Hence we obtain that if $\gamma\left(t_{1}\right)$ is an inflection point, then $\lim _{t \rightarrow t_{1}-0} N(t)=-\lim _{t \rightarrow t_{1}+0} N(t)$ and $\lim _{t \rightarrow t_{1}-0} B(t)=-\lim _{t \rightarrow t_{1}+0} B(t)$. Also, the torsion is equal to $\tau=v a$ except at the inflection points.

Now, in the case of $\mathcal{M}=S^{3}$, that is, $G>0$, we introduce the following parameters $\beta$, $\eta, p$ and $w$ defined by

$$
\beta=\frac{\alpha_{3}}{G}, \quad \eta=\frac{a_{+}}{\sqrt{\alpha_{3}}}, \quad p=\sqrt{\frac{\alpha_{3}-\alpha_{2}}{\alpha_{3}+\alpha_{1}}}, \quad w=\sqrt{\frac{\alpha_{3}}{\alpha_{3}+\alpha_{1}}} .
$$

Then,

Also,

$$
q=\frac{p}{w}, \quad y=\frac{\sqrt{G \beta}}{2 w} .
$$

$$
a_{+}=\eta \sqrt{G \beta}, \quad \alpha_{1}=\frac{G \beta\left(1-w^{2}\right)}{w^{2}}, \quad \alpha_{2}=\frac{G \beta\left(w^{2}-p^{2}\right)}{w^{2}}, \quad \alpha_{3}=G \beta .
$$


Under the relations (3.18) and (3.19), $\tilde{\mathcal{D}}$ has a one-to-one correspondence with

$$
\tilde{\mathcal{P}}=\left\{(\beta, \eta, p, w) \in \boldsymbol{R}^{4} ; \beta>0,0 \leqslant p \leqslant w \leqslant 1, w>0\right\} .
$$

We set $\mathcal{P}=\tilde{\mathcal{P}} / \sim$, where the equivalence relation $\sim$ is defined as follows: If $p=w$ or $w=1$, then $(\beta, \eta, p, w) \sim(\beta,-\eta, p, w)$. Then, $\mathcal{P}$ is in one-to-one correspondence with $\mathcal{D}$.

In Sections 6, 7 and 8 , we often use the space $\mathcal{P}$ rather than $\mathcal{D}$. We note that $\gamma$ is a helix if and only if $p=0$, and $\gamma$ has inflection points if and only if $p=w \neq 1$. Also, $k$ is not periodic if and only if $p=1(\Leftrightarrow p=w=1)$. The case of $p=1$ is called the Hasimoto soliton case (see the last paragraph of Section 4).

4. Construction of Killing vector fields. Let $\{\gamma, M\}$ be a Kirchhoff elastic rod in $\mathcal{M}=S^{3}, H^{3}$ or $\boldsymbol{R}^{3}$. (In what follows after Proposition 4.3, we assume $\mathcal{M}=S^{3}$.) In this section, we construct some Killing vector fields associated to $\{\gamma, M\}$, which play an essential role in Sections 5 and 6 . Unless otherwise specified, we assume, in the rest of the paper, that $t_{0}$ in (3.6) is zero, and the orientation of the frame $\left(T, M_{1}, M_{2}\right)$ is positive.

Let $J$ and $H$ be the vector fields along $\gamma$ defined by

$$
\begin{gathered}
J=2\left(\nabla_{T}\right)^{2} T+\left(3 k^{2}-\mu+2 v a^{2}\right) T-4 v a T \times \nabla_{T} T, \\
H=2 v a T+T \times \nabla_{T} T .
\end{gathered}
$$

Since $\gamma$ is real analytic, so are $J$ and $H$. We have the following proposition. (In the case of $G=0$, see [9], [20]. In the case of $v=0$, see [17], [15].)

Proposition 4.1. The vector fields $J, H$ along $\gamma$ are uniquely extended to Killing vector fields on $\mathcal{M}$.

To prove this, we need the following lemma, whose proof is omitted.

LEMma 4.2 ([17], [15]). Let $\mathcal{M}$ be $\boldsymbol{R}^{3}, S^{3}$ or $H^{3}$ of constant sectional curvature $G$. Let $\gamma=\gamma(t)$ be a unit-speed $C^{\infty}$ curve in $\mathcal{M}$ whose curvature $k(t)$ is positive everywhere. Let $\Lambda$ be a $C^{\infty}$ vector field along $\gamma$. Then $\Lambda$ can be extended to a Killing vector field on $\mathcal{M}$ if and only if $\Lambda$ satisfies the following system of differential equations.

$$
\begin{gathered}
\left\langle\nabla_{T} \Lambda, T\right\rangle=0, \\
\left\langle\left(\nabla_{T}\right)^{2} \Lambda+G \Lambda, N\right\rangle=0, \\
\left\langle\left(\nabla_{T}\right)^{3} \Lambda-\frac{k^{\prime}}{k}\left(\nabla_{T}\right)^{2} \Lambda+\left(G+k^{2}\right) \nabla_{T} \Lambda-\frac{k^{\prime}}{k} G \Lambda, B\right\rangle=0,
\end{gathered}
$$

where $(T, N, B)$ is the Frenet frame along $\gamma$. Moreover, the Killing vector field is uniquely determined. (Such a vector field $\Lambda$ is said to be a Killing vector field along $\gamma$.)

Proof of Proposition 4.1. Since $\gamma, J$ and $H$ are real analytic, it suffices to show that $J$ and $H$ satisfy (4.1), (4.2) and (4.3) on an open interval where $k>0$. By using the 
Frenet frame, $J, H$ are expressed as follows:

$$
J=\left(k^{2}-\mu+2 v a^{2}\right) T+2 k^{\prime} N+2 k(\tau-2 v a) B,
$$

$$
H=2 v a T+k B .
$$

By (2.4), $\nabla_{T}(J+2 G T)=0$. Thus,

$$
\nabla_{T} J=-2 G k N, \quad\left(\nabla_{T}\right)^{2} J=-2 G\left(-k^{2} T+k^{\prime} N+k \tau B\right) .
$$

Therefore we see that $J$ satisfies (4.1) and (4.2). Also,

$$
\left\langle\left(\nabla_{T}\right)^{3} J, B\right\rangle=-2 G\left(2 k^{\prime} \tau+k \tau^{\prime}\right),
$$

from which we have

$$
\left\langle\left(\nabla_{T}\right)^{3} J-\frac{k^{\prime}}{k}\left(\nabla_{T}\right)^{2} J+\left(G+k^{2}\right) \nabla_{T} J-\frac{k^{\prime}}{k} G J, B\right\rangle=\frac{-2 G}{k}\left[k^{2}(\tau-v a)\right]^{\prime} .
$$

By (3.10), the right hand side of the above expression is equal to zero. Therefore $J$ satisfies (4.3). It is easy to verify that $H$ satisfies (4.1). Also,

$$
\left\langle\left(\nabla_{T}\right)^{2} H, N\right\rangle=-\frac{1}{k}\left[k^{2}(\tau-v a)\right]^{\prime}=0 .
$$

Hence $H$ satisfies (4.2). By (3.9), we have

$$
\left\langle\left(\nabla_{T}\right)^{3} H, B\right\rangle=-\frac{1}{2}\left[k^{3}+\left(2 v a^{2}-(\mu-2 G)\right) k\right]^{\prime},
$$

from which follows that

$$
\begin{aligned}
& \left\langle\left(\nabla_{T}\right)^{3} H-\frac{k^{\prime}}{k}\left(\nabla_{T}\right)^{2} H+\left(G+k^{2}\right) \nabla_{T} H-\frac{k^{\prime}}{k} G H, B\right\rangle \\
& =\frac{-k^{\prime}}{2 k}\left[2 k^{\prime \prime}+k^{3}+\left(2 v a^{2}-(\mu-2 G)\right) k-2 k \tau(\tau-2 v a)\right]=0 .
\end{aligned}
$$

Therefore $H$ satisfies (4.3).

Let $\tilde{J}$ and $\tilde{H}$ denote the unique extensions of $J$ and $H$ as Killing vector fields on $\mathcal{M}$. If $\mathcal{M}=\boldsymbol{R}^{3}$, then we see, with a few exceptions, that $\tilde{J}$ is a non-zero constant vector filed and $\tilde{H}$ is a Killing vector field corresponding to a screw motion around an axis parallel to $\tilde{J}$, so that we can construct the cylindrical coordinates associated to $\tilde{J}$ and $\tilde{H}$ (cf. [9], [20]).

From now on, we always assume $\mathcal{M}=S^{3}$. Let $I_{+}$and $I_{-}$be the vector fields along $\gamma$ defined by

$$
I_{+}=J+2 \sqrt{G} H, \quad I_{-}=J-2 \sqrt{G} H .
$$

PROPOSITION 4.3. The vector fields $I_{+}$and $I_{-}$along $\gamma$ are uniquely extended to Killing vector fields on $S^{3}$.

PROOF. It suffices to show that $I_{+}$and $I_{-}$satisfy (4.1), (4.2) and (4.3) on an open interval where $k>0$. Since $J$ and $H$ satisfy (4.1), (4.2) and (4.3) and these differential equations are linear, $I_{+}$and $I_{-}$also satisfy these equations. 
Let $\tilde{I}_{+}$and $\tilde{I}_{-}$denote the unique extensions of $I_{+}$and $I_{-}$as Killing vector fields on $S^{3}$. By using $\tilde{J}, \tilde{H}, \tilde{I}_{+}$and $\tilde{I}_{-}$, we construct an appropriate system of local coordinates in Section 5. Now, we show that $J, H, I_{+}$and $I_{-}$have the following property.

Proposition 4.4. The functions $\langle J, H\rangle,\left|I_{+}\right|$and $\left|I_{-}\right|$on $\boldsymbol{R}$ are constant.

PROOF. Since these functions are real analytic, it suffices to show that they are constant on an open interval where $k>0$. By (4.4), (4.5) and (3.10), we have

$$
\langle J, H\rangle=2 b+2\left(2 v a^{2}-\mu\right) v a=2 d=\text { const. },
$$

where we set

$$
d=b+\left(2 v a^{2}-\mu\right) v a .
$$

Also, by (3.10) and (3.11),

$$
|J|^{2}=\left(4 c-4 G k^{2}\right)+\left(2 v a^{2}-\mu\right)^{2}-8 v a b .
$$

Thus we have

$$
\begin{aligned}
\left|I_{ \pm}\right|^{2} & =|J|^{2}+4 G|H|^{2} \pm 4 \sqrt{G}\langle J, H\rangle \\
& =4 c+\left(2 v a^{2}-\mu\right)^{2}-8 v a b+16 G v^{2} a^{2} \pm 8 d \sqrt{G}=\text { const. }
\end{aligned}
$$

In the rest of this section, we give a corollary of Proposition 4.1 relevant to the vortex filament equation. (In the following sections, there is no need to use this corollary.) We consider the following evolution equation for curves in a 3-dimensional oriented Riemannian manifold $\mathcal{M}$ :

$$
\frac{\partial \tilde{\gamma}}{\partial \lambda}=\frac{\partial \tilde{\gamma}}{\partial t} \times \nabla_{t} \frac{\partial \tilde{\gamma}}{\partial t}
$$

where $\tilde{\gamma}=\tilde{\gamma}(\lambda, t): \boldsymbol{R} \times \boldsymbol{R} \rightarrow \mathcal{M}$ and $\lambda$ denotes the time and $t$ the parameter along the curve. The equation (4.8) is known as the vortex filament equation ([5], [6], [11], [14], [20]), which is an idealized model of the evolution of vortex filaments in three dimensional inviscid incompressible fluids. (This equation is also called the localized induction equation or the Betchov-Da Rios equation.)

The definition of $H$ and an easy calculation yield the following corollary, which extends Corollary 4 of [20] to 3-dimensional space forms $\boldsymbol{R}^{3}, S^{3}, H^{3}$.

COROLlaRY 4.5. Let $\mathcal{M}=\boldsymbol{R}^{3}, S^{3}$ or $H^{3}$. Let $\gamma$ be the centerline of a Kirchhoff elastic rod in $\mathcal{M}$ with twist rate a. Then, $\tilde{\gamma}(\lambda, t):=(\operatorname{Exp} \lambda \tilde{H}) \circ \gamma(t-2 v a \lambda)$ satisfies the equation (4.8), where $\operatorname{Exp} \lambda \tilde{H}(\lambda \in \boldsymbol{R})$ is the one-parameter group of isometries on $\mathcal{M}$ generated by the Killing vector filed $\tilde{H}$.

Therefore, the centerline $\gamma$ of a Kirchhoff elastic rod evolves under the equation (4.8) without changing shape. When $p=1$, the shape of $\gamma$ is of the soliton type. In the case of $\mathcal{M}=\boldsymbol{R}^{3}$, this soliton solution was discovered by Hasimoto in [6] (see also [11]). For this reason, the case of $p=1$ is called the Hasimoto soliton case. 
5. Construction of the coordinates. In this section, we construct a local coordinate system associated to the Killing vector fields $\tilde{J}, \tilde{H}, \tilde{I}_{+}$and $\tilde{I}_{-}$. We embed $S^{3}$ isometrically into the 4-dimensional Euclidean space $\boldsymbol{R}^{4}$ as the standard sphere of radius $1 / \sqrt{G}$ :

$$
S^{3}=S^{3}(1 / \sqrt{G})=\left\{{ }^{t}\left(x_{1}, x_{2}, x_{3}, x_{4}\right) \in \boldsymbol{R}^{4} ; x_{1}^{2}+x_{2}^{2}+x_{3}^{2}+x_{4}^{2}=1 / G\right\} .
$$

Let $\iota: S^{3} \hookrightarrow \boldsymbol{R}^{4}$ denote the inclusion map. We denote the Euclidean inner product on $\boldsymbol{R}^{4}$ and the Riemannian metric on $S^{3}$ by the same notation $\langle$,$\rangle .$

For such an embedding $\iota$, we take a local coordinate system $(r, \theta, \psi)$ in $S^{3}$ satisfying the following relations:

$$
x_{1}=r \cos \theta, \quad x_{2}=r \sin \theta, \quad x_{3}=\bar{r} \cos \psi, \quad x_{4}=\bar{r} \sin \psi,
$$

where $r>0$ and

$$
\bar{r}=\sqrt{1 / G-r^{2}} .
$$

Note that each level surface of the coordinate $r$ is a Clifford torus. (When $r=0$ or $1 / \sqrt{G}$, it degenerates to a great circle.) The coordinate vector fields $\partial / \partial r, \partial / \partial \theta, \partial / \partial \psi$ are orthogonal, and the lengths of these are given by

$$
\left|\frac{\partial}{\partial r}\right|=\frac{1}{\sqrt{G} \bar{r}}, \quad\left|\frac{\partial}{\partial \theta}\right|=r, \quad\left|\frac{\partial}{\partial \psi}\right|=\bar{r} .
$$

Now, for a Killing vector field $Y$ on $S^{3}$, there exists a unique skew symmetric $4 \times 4$ matrix $A$, called the matrix representation of the Killing vector field $Y$ with respect to $\iota$, satisfying

$$
Y(\boldsymbol{x})=A \boldsymbol{x}, \quad \boldsymbol{x}={ }^{t}\left(x_{1}, x_{2}, x_{3}, x_{4}\right) \in S^{3} .
$$

It should be noted that $\partial / \partial \theta, \partial / \partial \psi$ are naturally extended to the Killing vector fields on $S^{3}$ represented by the following skew symmetric matrices $E_{1}, E_{2}$, respectively.

$$
E_{1}=\left(\begin{array}{cccc}
0 & -1 & 0 & 0 \\
1 & 0 & 0 & 0 \\
0 & 0 & 0 & 0 \\
0 & 0 & 0 & 0
\end{array}\right), \quad E_{2}=\left(\begin{array}{cccc}
0 & 0 & 0 & 0 \\
0 & 0 & 0 & 0 \\
0 & 0 & 0 & -1 \\
0 & 0 & 1 & 0
\end{array}\right)
$$

We denote the extensions of $\partial / \partial \theta, \partial / \partial \psi$ to $S^{3}$ by the same notation.

In what follows, we give an appropriate $P \in O(4)$ and construct $(r, \theta, \psi)$ with respect to the embedding $P \circ \iota$. First, we consider the case where the parameter $p>0$.

Lemma 5.1. Suppose that $p>0$. Then the functions $\langle\tilde{J}, \tilde{H}\rangle,\left|\tilde{I}_{+}\right|,\left|\tilde{I}_{-}\right|$on $S^{3}$ are constant.

PROOF. Seeking a contradiction, we suppose that $\left|\tilde{I}_{+}\right|$is not constant on $S^{3}$. Let $A$ be the matrix representation of $\tilde{I}_{+}$. Then there exists $P \in O(4)$ such that $P A P^{-1}$ is of the canonical form, that is, $P A P^{-1}=\sigma_{1} E_{1}+\sigma_{2} E_{2}$, where $\sigma_{1}, \sigma_{2}$ are real constants. Hence the matrix representation of $\tilde{I}_{+}$with respect to $P \circ \iota$ is $\sigma_{1} E_{1}+\sigma_{2} E_{2}$. We rewrite $P \circ \iota$ as $\iota$. By the assumption $\left|\tilde{I}_{+}\right| \not \equiv$ const., we see $\left|\sigma_{1}\right| \neq\left|\sigma_{2}\right|$. 
Let $r(t), \theta(t), \psi(t)$ denote the $r, \theta, \psi$ components of $\gamma(t)$. Then,

$$
\left|I_{+}(t)\right|^{2}=\sigma_{1}^{2} r(t)^{2}+\sigma_{2}^{2} \bar{r}(t)^{2}=r(t)^{2}\left(\sigma_{1}^{2}-\sigma_{2}^{2}\right)+\sigma_{2}^{2} / G,
$$

from which we obtain

$$
r(t)^{2}=\frac{\left|I_{+}(t)\right|^{2}-\left(\sigma_{2}^{2} / G\right)}{\sigma_{1}^{2}-\sigma_{2}^{2}} .
$$

Therefore, by Proposition 4.4, $r(t)$ is independent of $t$. If $r=0$ or $1 / \sqrt{G}$, then $\gamma$ is a great circle, that is, a geodesic. Thus, $r \neq 0,1 / \sqrt{G}$. Hence $\gamma$ lies on the Clifford torus $\left\{\boldsymbol{x} \in S^{3} ; x_{1}^{2}+x_{2}^{2}=r^{2}\right\}$.

It is easy to verify the following formulas:

$$
\begin{aligned}
& \nabla_{\partial / \partial \theta} \partial / \partial \theta=-G r \bar{r}^{2} \partial / \partial r, \quad \nabla_{\partial / \partial \theta} \partial / \partial \psi=\nabla_{\partial / \partial \psi} \partial / \partial \theta=0, \\
& \nabla_{\partial / \partial \psi} \partial / \partial \psi=G r \bar{r}^{2} \partial / \partial r .
\end{aligned}
$$

By using these formulas and calculating the components of $\nabla_{T} T$ with respect to the frame $(\partial / \partial r, \partial / \partial \theta, \partial / \partial \psi)$, we see that the squared curvature $u(t)$ is expressed as

$$
u(t)=G r^{2} \bar{r}^{2}\left(\left(\theta^{\prime}\right)^{2}-\left(\psi^{\prime}\right)^{2}\right)^{2}+\left(\theta^{\prime \prime}\right)^{2} r^{2}+\left(\psi^{\prime \prime}\right)^{2} \bar{r}^{2} .
$$

Note that (5.1) holds for a general unit-speed curve lying on the above Clifford torus.

By using the fact that $\{\gamma, M\}$ is a Kirchhoff elastic rod, we are going to show that $\theta^{\prime}(t)$, $\psi^{\prime}(t), \theta^{\prime \prime}(t)$ and $\psi^{\prime \prime}(t)$ are expressed in terms of $u(t)$, and derive an equation of $u(t)$. First, we show the following

LEMMA 5.2 .

$$
\begin{aligned}
& \theta^{\prime}(t)=\frac{\left(u(t)-\mu+2 v a^{2}+4 \sqrt{G} v a\right) \sigma_{1} r \pm \sigma_{2} \bar{r} \sqrt{D(u(t))}}{r\left(\sigma_{1}^{2} r^{2}+\sigma_{2}^{2} \bar{r}^{2}\right)}, \\
& \psi^{\prime}(t)=\frac{\left(u(t)-\mu+2 v a^{2}+4 \sqrt{G} v a\right) \sigma_{2} \bar{r} \mp \sigma_{1} r \sqrt{D(u(t))}}{\bar{r}\left(\sigma_{1}^{2} r^{2}+\sigma_{2}^{2} \bar{r}^{2}\right)},
\end{aligned}
$$

where $D(u)=-\left(u-\mu+2 v a^{2}+4 \sqrt{G} v a\right)^{2}+\sigma_{1}^{2} r^{2}+\sigma_{2}^{2} \bar{r}^{2}$.

Proof. Since $r(t)$ is constant, $T=\theta^{\prime}(\partial / \partial \theta)+\psi^{\prime}(\partial / \partial \psi)$. Thus, by $|T| \equiv 1$, we see

$$
r^{2}\left(\theta^{\prime}\right)^{2}+\bar{r}^{2}\left(\psi^{\prime}\right)^{2}=1 \text {. }
$$

We now calculate $\left\langle I_{+}, T\right\rangle$ in two ways. By (4.4) and (4.5), we have

$$
\left\langle I_{+}, T\right\rangle=u(t)-\mu+2 v a^{2}+4 \sqrt{G} v a
$$

on an open interval where $k>0$. Because of the real analyticity of $\left\langle I_{+}, T\right\rangle$, this expression is valid for the whole $\boldsymbol{R}$. On the other hand, it follows from $\tilde{I}_{+}=\sigma_{1}(\partial / \partial \theta)+\sigma_{2}(\partial / \partial \psi)$ that $\left\langle I_{+}, T\right\rangle=\sigma_{1} r^{2} \theta^{\prime}+\sigma_{2} \bar{r}^{2} \psi^{\prime}$. Thus,

$$
\sigma_{1} r^{2} \theta^{\prime}+\sigma_{2} \bar{r}^{2} \psi^{\prime}=u(t)-\mu+2 v a^{2}+4 \sqrt{G} v a .
$$

Note that $D(u(t))=-\left\langle I_{+}, T\right\rangle^{2}+\left|I_{+}\right|^{2} \geqslant 0$. Hence, by (5.4) and (5.5), we obtain (5.2) and (5.3) 
We proceed with the proof of Lemma 5.1. Let $W$ be an open interval such that $u(t) \neq \alpha_{3}$, $\alpha_{2}$ for all $t \in W$. If $D(u(t))=0$, then $I_{+}(t)$ is parallel to $T(t)$, and $k^{\prime}(t)=0$ by (4.4) and (4.5), so that $u(t)=\alpha_{3}$ or $\alpha_{2}$. Therefore, $D(u(t))>0$ on $W$. We assume that in (5.2) and (5.3), the upper sign is taken. (The proof is similar, when the lower sign is taken.) In what follows, we restrict the domain of $t$ to $W$.

By Lemma 5.2 and (3.12), we have

$$
\begin{aligned}
& \left(\theta^{\prime \prime}(t)\right)^{2}=\frac{1}{r^{2}\left(\sigma_{1}^{2} r^{2}+\sigma_{2}^{2} \bar{r}^{2}\right)^{2}}\left[\sigma_{1} r-\frac{\sigma_{2} \bar{r}\left(u(t)-\mu+2 v a^{2}+4 \sqrt{G} v a\right)}{\sqrt{D(u(t))}}\right]^{2} Q(u(t)), \\
& \left(\psi^{\prime \prime}(t)\right)^{2}=\frac{1}{\bar{r}^{2}\left(\sigma_{1}^{2} r^{2}+\sigma_{2}^{2} \bar{r}^{2}\right)^{2}}\left[\sigma_{2} \bar{r}+\frac{\sigma_{1} r\left(u(t)-\mu+2 v a^{2}+4 \sqrt{G} v a\right)}{\sqrt{D(u(t))}}\right]^{2} Q(u(t)),
\end{aligned}
$$

where $Q(u)$ is the right hand side of (3.12). Let $\mathcal{F}(u)$ be the expression obtained by substituting (5.2), (5.3), (5.6) and (5.7) to the right hand side of (5.1). Then we have

$$
u(t)=\mathcal{F}(u(t)) \text { for all } t \in W .
$$

Moreover, the function $\mathcal{F}(u)$ has the following property.

Lemma 5.3. Let $V$ denote the open interval defined by $V=\{u \in \boldsymbol{R} ; D(u)>0\}$. Then the function $\mathcal{F}(u)-u$ is real analytic on $V$ and not identically zero. Consequently, any zero of $\mathcal{F}(u)-u$ in $V$ is isolated.

Proof. We set $\mathcal{G}(u)=\mathcal{F}(u)-u$. It is easy to see that $\mathcal{G}$ is real analytic on $V$. By (5.2), (5.3), (5.6) and (5.7), we obtain

$$
\frac{\mathcal{G}(u)}{u^{4}}=\frac{r^{2}}{G \bar{r}^{2}}\left(\frac{\left(\theta^{\prime}\right)^{2}-G}{u^{2}}\right)^{2}+\frac{\left(\theta^{\prime \prime}\right)^{2} r^{2}+\left(\psi^{\prime \prime}\right)^{2} \bar{r}^{2}}{u^{4}}-\frac{1}{u^{3}} \rightarrow \frac{\left(\sigma_{1} r \pm \sigma_{2} \bar{r} \sqrt{-1}\right)^{4}}{G r^{2} \bar{r}^{2}\left(\sigma_{1}^{2} r^{2}+\sigma_{2}^{2} \bar{r}^{2}\right)^{4}} \neq 0
$$

as $u(\in \boldsymbol{R}) \rightarrow+\infty$. (Here, the \pm sign is determined up to the choice of the branch of $\sqrt{D(u)}$.) Therefore, $\mathcal{G}$ is not identically zero on $V$.

On the other hand, by (5.8) together with the assumption that $\gamma$ is not a helix, the set of all roots of the equation $u=\mathcal{F}(u) \quad(u \in V)$ contains some open interval in $\boldsymbol{R}$. This contradicts Lemma 5.3. Therefore, $\left|\tilde{I}_{+}\right|$is constant on the whole $S^{3}$.

Similarly, we can see that $\left|\tilde{I}_{-}\right|$is constant on $S^{3}$. Since $\langle\tilde{J}, \tilde{H}\rangle=\left(\left|\tilde{I}_{+}\right|^{2}-\left|\tilde{I}_{-}\right|^{2}\right) /(8 \sqrt{G})$, it follows that $\langle\tilde{J}, \tilde{H}\rangle$ is also constant on $S^{3}$. This completes the proof of Lemma 5.1.

Proposition 5.4. Suppose that $p>0$, that is, $\gamma$ is not a helix. Then, there exist $P \in O(4)$ and positive numbers $f, g$ such that the matrix representations of $\tilde{I}_{+}$and $\tilde{I}_{-}$with respect to the isometric embedding $P \circ \iota: S^{3} \rightarrow \boldsymbol{R}^{4}$ are expressed as $f E_{1}+f E_{2}$ and $-g E_{1}+g E_{2}$, respectively.

Proof. Let $A_{\tilde{J}}, A_{\tilde{H}}, A_{\tilde{I}_{+}}, A_{\tilde{I}_{-}}$denote the matrix representations of $\tilde{J}, \tilde{H}, \tilde{I}_{+}, \tilde{I}_{-}$, respectively. Without loss of generality, we may assume that $A_{\tilde{J}}=\rho_{1} E_{1}+\rho_{2} E_{2}$, where $\rho_{1}$, $\rho_{2}$ are real constants. By (4.7) together with the assumption that $\gamma$ is not a helix, $|J(t)|^{2}$ is not constant, so that $\left|\rho_{1}\right| \neq\left|\rho_{2}\right|$. 
We show that $A_{\tilde{H}}, A_{\tilde{I}_{+}}$and $A_{\tilde{I}_{-}}$are also canonical forms. Let

$$
A_{\tilde{H}}=\left(\begin{array}{cccc}
0 & -h_{1} & -h_{2} & -h_{3} \\
h_{1} & 0 & -h_{4} & -h_{5} \\
h_{2} & h_{4} & 0 & -h_{6} \\
h_{3} & h_{5} & h_{6} & 0
\end{array}\right),
$$

where $h_{1}, \ldots, h_{6} \in \boldsymbol{R}$. By Lemma 5.1 and (4.6), we have $\left\langle A_{\tilde{J}} \boldsymbol{x}, A_{\tilde{H}} \boldsymbol{x}\right\rangle=2 d G\langle\boldsymbol{x}, \boldsymbol{x}\rangle$ for all $\boldsymbol{x} \in \boldsymbol{R}^{4}$. Therefore,

$$
{ }^{t} A_{\tilde{J}} A_{\tilde{H}}+{ }^{t}\left({ }^{t} A_{\tilde{J}} A_{\tilde{H}}\right)=(4 d G) E,
$$

where $E$ is the identity matrix. Thus we have

$$
\left(\begin{array}{ll}
\rho_{2} & \rho_{1} \\
\rho_{1} & \rho_{2}
\end{array}\right)\left(\begin{array}{cc}
h_{3} & h_{5} \\
-h_{4} & h_{2}
\end{array}\right)=0
$$

Therefore, by $\left|\rho_{1}\right| \neq\left|\rho_{2}\right|$, we obtain $h_{2}=h_{3}=h_{4}=h_{5}=0$. Hence $A_{\tilde{H}}$ is a canonical form. Consequently, $A_{\tilde{I}_{+}}, A_{\tilde{I}_{-}}$are also canonical forms, because $\tilde{I}_{+}, \tilde{I}_{-}$are linear combinations of $\tilde{J}, \tilde{H}$.

Let $A_{\tilde{I}_{+}}=f_{1} E_{1}+f_{2} E_{2}$, where $f_{1}, f_{2} \in \boldsymbol{R}$. By Lemma 5.1, $\left|f_{1}\right|=\left|f_{2}\right|$. Thus, there exists an orthogonal transformation $P_{1}$ on $\boldsymbol{R}^{4}$ permuting the coordinates $x_{1}, x_{2}, x_{3}, x_{4}$ such that the matrix representation of $\tilde{I}_{+}$with respect to $P_{1} \circ \iota$ is expressed as $f E_{1}+f E_{2}$, where $f=\left|f_{1}\right|=\left|f_{2}\right|$. If $f=0$, then $I_{+} \equiv 0$, which contradicts the assumption that $\gamma$ is not a helix. Hence $f>0$.

By Lemma 5.1, the matrix representation of $\tilde{I}_{-}$with respect to $P_{1} \circ \iota$ is $g E_{1}+g E_{2}$ or $-g E_{1}+g E_{2}$, where $g$ is a non-zero constant. In the case of $g E_{1}+g E_{2}$, we see $f I_{-}=g I_{+}$. Thus, by comparing the $N$ components of the both sides, we have $f=g$, and hence $I_{+}=I_{-}$. Therefore, $H=0$, which contradicts the assumption that $\gamma$ is not a geodesic. Consequently, the matrix representation of $\tilde{I}_{-}$is $-g E_{1}+g E_{2}$. Hence, in the case of $g>0$, it suffices to set $P=P_{1}$. In the case of $g<0$, it suffices to set $P=P_{2} \circ P_{1}$, where $P_{2} \in O(4)$ is the transformation sending ${ }^{t}\left(x_{1}, x_{2}, x_{3}, x_{4}\right)$ to ${ }^{t}\left(x_{3}, x_{4}, x_{1}, x_{2}\right)$, and to rewrite $-g$ as $g$.

Next, we consider the case of $p=0$.

Proposition 5.5. Suppose that $p=0$, that is, $\gamma$ is a helix. Then $T$ is uniquely extended to a Killing vector filed $\tilde{T}$ on $S^{3}$, and the following holds. There exist $P \in O$ (4) and real numbers $\sigma$ and $\rho$ such that $\rho \geqslant|\sigma|$, the matrix representation of $\tilde{T}$ with respect to the isometric embedding $P \circ \iota: S^{3} \rightarrow \boldsymbol{R}^{4}$ is expressed as $\sigma E_{1}+\rho E_{2}$, and the orientation of the frame $(\partial / \partial r, \partial / \partial \theta, \partial / \partial \psi)$ with respect to $P \circ \iota$ is negative.

PROOF. It is verified by a straightforward calculation that $T$ is a Killing vector field along $\gamma$. Therefore, $T$ is uniquely extended to a Killing vector filed $\tilde{T}$ on $S^{3}$. Hence there exists $P_{1} \in O(4)$ and real numbers $\sigma$ and $\rho$ such that $\rho \geqslant|\sigma|$ and the matrix representation of $\tilde{T}$ with respect to $P_{1} \circ \iota$ is expressed as $\sigma E_{1}+\rho E_{2}$. 
If the orientation of $(\partial / \partial r, \partial / \partial \theta, \partial / \partial \psi)$ with respect to $P_{1} \circ \iota$ is negative, it suffices to set $P=P_{1}$. Otherwise, it suffices to set $P=P_{2} \circ P_{1}$, where $P_{2} \in O(4)$ is the transformation ${ }^{t}\left(x_{1}, x_{2}, x_{3}, x_{4}\right) \mapsto{ }^{t}\left(x_{2}, x_{1}, x_{3}, x_{4}\right)$, and to rewrite $-\sigma$ as $\sigma$.

6. Explicit solutions in terms of elliptic functions and integrals. In this section, we give the explicit expressions of the $r, \theta, \psi$ components of $\gamma$ in terms of the parameters $\beta, \eta$, $p, w$, and Jacobi sn function and the incomplete elliptic integral of the third kind (Theorem 6.1). We also show that the $\partial / \partial r, \partial / \partial \theta, \partial / \partial \psi$ components of $M$ are explicitly expressed.

First, we express various constants in the previous sections by $(\beta, \eta, p, w)(\in \tilde{\mathcal{P}})$. In order to simplify the expressions of $\beta, \eta, p$ and $w$, we introduce the following notation:

$$
\begin{aligned}
& V=\sqrt{1-w^{2}}, \quad X=\sqrt{w^{2}-p^{2}}, \quad Y_{1}=1+p^{2}-\left(1+4 v^{2} \eta^{2}\right) w^{2}-4 w^{2} / \beta, \\
& R=V X-2 v \eta w^{2}, \quad Y_{2}=Y_{1}-4 v \eta R, \quad Z=\sqrt{Y_{1}^{2}+4 R^{2}}, \\
& \Omega=V / 2 w+v \eta, \quad \Gamma=V / 2 w-v \eta, \quad U=-\beta Z^{2}+16 w^{4}\left(1+4 v^{2} \eta^{2}\right), \\
& \Lambda=\sqrt{64 \beta w^{4} Y_{2}^{2}+U^{2}}, \quad S_{1}=X\left(1-p^{2}-\left(1-4 v^{2} \eta^{2}+4 / \beta\right) w^{2}\right)-4 v \eta w^{2} V .
\end{aligned}
$$

Then it is verified by a straightforward calculation that the following equalities hold.

$$
\begin{gathered}
Z^{2}\left(1+4 v^{2} \eta^{2}\right)-4\left(v \eta Y_{1}+R\right)^{2}=Y_{2}^{2}, \\
w^{2} Y_{2}^{2}-p^{2} Z^{2}+\frac{16 p^{2} w^{2}}{\beta}\left(X^{2}+4 v^{2} \eta^{2} w^{2}\right)=S_{1}^{2}, \\
\Lambda=\sqrt{64 \beta w^{2} S_{1}^{2}+\left(U-32 p^{2} w^{2}\right)^{2}} .
\end{gathered}
$$

We express $a, b, \mu, f$ and $g$ by $(\beta, \eta, p, w)$. In the rest of the paper, the upper sign is taken when $b \geqslant 0$, while the lower sign is taken when $b<0$. By (3.13), (3.14), (3.15) and (3.19), we have

$$
\begin{gathered}
a= \pm a_{+}= \pm \eta \sqrt{G \beta}, \\
b= \pm \frac{G^{3 / 2} \beta^{3 / 2}}{2 w^{2}} V X \\
\mu=\frac{G \beta}{2 w^{2}}\left[-Y_{1}+2 w^{2}\left(1+2 v \eta^{2}\right)\right] .
\end{gathered}
$$

By Proposition 5.4, we have $\left|\tilde{I}_{+}(\boldsymbol{x})\right|^{2}=f^{2}|\boldsymbol{x}|^{2}=f^{2} / G$, where $\boldsymbol{x} \in S^{3}$. Thus, $f=$ $\sqrt{G}\left|\tilde{I}_{+}\right|=\sqrt{G}\left|I_{+}(0)\right|$. Since

$$
I_{+}(0)=\left(\alpha_{3}-\mu+2 v a^{2}+4 v a \sqrt{G}\right) T(0)+2 \sqrt{\alpha_{3}}\left(\left(b / \alpha_{3}\right)-v a+\sqrt{G}\right) B(0),
$$

a straightforward calculation using (6.4), (6.5) and (6.6) yields

$$
f=\frac{G^{3 / 2} \beta^{1 / 2}}{2 w^{2}} \sqrt{\left(\beta^{1 / 2} Y_{1} \pm 8 v \eta w^{2}\right)^{2}+4\left(\beta^{1 / 2} R \pm 2 w^{2}\right)^{2}} .
$$


In the same way, we see $g=\sqrt{G}\left|\tilde{I}_{-}\right|=\sqrt{G}\left|I_{-}(0)\right|$, and by a similar calculation, obtain

$$
g=\frac{G^{3 / 2} \beta^{1 / 2}}{2 w^{2}} \sqrt{\left(\beta^{1 / 2} Y_{1} \mp 8 v \eta w^{2}\right)^{2}+4\left(\beta^{1 / 2} R \mp 2 w^{2}\right)^{2}} .
$$

To state the first main theorem, we introduce the following notation:

$$
\begin{aligned}
& \xi=\sqrt{\frac{32 p^{2} w^{2}}{\Lambda+U}, \quad \zeta=\sqrt{\frac{32 p^{2} w^{2}}{\Lambda-U}}} \\
& D_{1}=\frac{\beta\left[Y_{1}-2 w^{2}\left(1+4 v^{2} \eta^{2}\right)\right]}{w^{2}}\left(\frac{f-g}{G^{3 / 2}}\right)+\frac{(f-g)^{3}}{8 G^{9 / 2}} \mp \frac{8 v \eta \beta^{1 / 2}(f+g)}{G^{3 / 2}}, \\
& D_{2}=\frac{\beta\left[Y_{1}-2 w^{2}\left(1+4 v^{2} \eta^{2}\right)\right]}{w^{2}}\left(\frac{f+g}{G^{3 / 2}}\right)+\frac{(f+g)^{3}}{8 G^{9 / 2}} \mp \frac{8 v \eta \beta^{1 / 2}(f-g)}{G^{3 / 2}}, \\
& C_{1}=\frac{-4 w^{5} D_{1}}{\beta^{3 / 2}(\Lambda+U)}, \quad C_{2}=\frac{4 w^{5} D_{2}}{\beta^{3 / 2}(\Lambda-U)}, \\
& Q_{1}=\sqrt{\beta+( \pm \sqrt{\beta} \Omega-1)^{2}}, \quad Q_{2}=\sqrt{\beta+(\mp \sqrt{\beta} \Omega-1)^{2}} .
\end{aligned}
$$

By the definition of $\Lambda$ and (6.3), we can check that if $p>0$, then $\Lambda+U>0$ and hence $\xi$ and $C_{1}$ are well-defined. Also, we see that $\Lambda-U \geqslant 0$, where the equality holds if and only if $Y_{2}=0$ and $U \geqslant 0$. Thus, except the case where $Y_{2}=0$ and $U \geqslant 0$ hold, $\zeta$ and $C_{2}$ are well-defined.

We denote by

$$
\Pi(x, \alpha, p)=\int_{0}^{x} \frac{d x}{1-\alpha \operatorname{sn}^{2}(x, p)}
$$

the incomplete elliptic integral of the third kind, where $\alpha, p$ are real numbers satisfying $\alpha \leqslant 1$, $0 \leqslant p \leqslant 1$. When $x=K(p)$, the integral is said to be complete.

THEOREM 6.1. Let $r(t), \theta(t), \psi(t)$ denote the $r, \theta, \psi$ components of $\gamma(t)$.

Case 1. The case of $p>0$.

(1) $r(t)$ is given by

$$
r(t)=\sqrt{\frac{U+\Lambda-32 p^{2} w^{2} \operatorname{sn}^{2}(y t, p)}{2 G \Lambda}} .
$$

(a) If $p=1$, then $r$ does not attain the minimum value. If $p \neq 1$, then the minimum value $r_{\min }$ of $r$ is attained, and the condition $r_{\min }=0$ is equivalent to

$$
S_{1}=0 \text { and } U-32 p^{2} w^{2} \leqslant 0 .
$$

(b) The maximum value $r_{\max }$ of $r$ is attained, and the condition $r_{\max }=1 / \sqrt{G}$ is equivalent to

$$
Y_{2}=0 \text { and } U \geqslant 0 \text {. }
$$

(2) $\theta(t)$ is given as follows: 
If (6.10) does not hold or $p=1$, then

$$
\theta(t)=\frac{-(f-g)}{8 G} t+C_{1} \Pi\left(y t, \xi^{2}, p\right)+\theta(0) .
$$

If (6.10) holds and $p \neq 1$, then

(6.13) $\theta(t)=\frac{-(f-g)}{8 G} t+m \pi+\theta(0), \quad \frac{(2 m-1) K(p)}{y}<t<\frac{(2 m+1) K(p)}{y}$,

where $m$ is an arbitrary integer.

(3) $\psi(t)$ is given as follows:

If (6.11) does not hold, then

$$
\psi(t)=\frac{-(f+g)}{8 G} t+C_{2} \Pi\left(y t,-\zeta^{2}, p\right)+\psi(0) .
$$

If (6.11) holds, then

$$
\begin{gathered}
\psi(t)=\frac{-(f+g)}{8 G}\left(t-\frac{K(p)}{y}\right)+m \pi+\psi\left(\frac{K(p)}{y}\right), \\
\frac{2 m K(p)}{y}<t<\frac{(2 m+2) K(p)}{y},
\end{gathered}
$$

where $m$ is an arbitrary integer.

Case 2. The case of $p=0$.

The constants $\sigma$ and $\rho$ in Proposition 5.5 are expressed as

$$
\sigma=\frac{\sqrt{G}\left(Q_{1}-Q_{2}\right)}{2}, \quad \rho=\frac{\sqrt{G}\left(Q_{1}+Q_{2}\right)}{2},
$$

and $r(t), \theta(t)$ and $\psi(t)$ are expressed as follows:

$$
\begin{gathered}
r(t)=\sqrt{\frac{1-\left(\rho^{2} / G\right)}{\sigma^{2}-\rho^{2}}}, \\
\theta(t)=\sigma t+\theta(0), \quad \psi(t)=\rho t+\psi(0) .
\end{gathered}
$$

ProOF. First, we consider Case 1. By Proposition 5.4, we have

$$
\begin{gathered}
\tilde{I}_{+}=f \frac{\partial}{\partial \theta}+f \frac{\partial}{\partial \psi}, \quad \tilde{I}_{-}=-g \frac{\partial}{\partial \theta}+g \frac{\partial}{\partial \psi}, \\
\tilde{J}=\left(\frac{f-g}{2}\right) \frac{\partial}{\partial \theta}+\left(\frac{f+g}{2}\right) \frac{\partial}{\partial \psi}, \\
\tilde{H}=\left(\frac{f+g}{4 \sqrt{G}}\right) \frac{\partial}{\partial \theta}+\left(\frac{f-g}{4 \sqrt{G}}\right) \frac{\partial}{\partial \psi} .
\end{gathered}
$$

Therefore,

$$
|H(t)|^{2}=|\tilde{H}(\gamma(t))|^{2}=\left(\frac{f+g}{4 \sqrt{G}}\right)^{2} r(t)^{2}+\left(\frac{f-g}{4 \sqrt{G}}\right)^{2}\left(\frac{1}{G}-r(t)^{2}\right) .
$$


On the other hand, by the definition of $H,|H(t)|^{2}=u(t)+4 v^{2} a^{2}$. Hence we obtain

$$
r(t)=\sqrt{\frac{1}{4 f g}\left[16 G\left(u(t)+4 v^{2} a^{2}\right)-\frac{(f-g)^{2}}{G}\right]} .
$$

A straightforward calculation using (6.7), (6.8) and (6.1) then yields

$$
2 f g=\frac{G^{3} \beta \Lambda}{2 w^{4}}, \quad 16 G\left(\alpha_{3}+4 v^{2} a^{2}\right)-\frac{(f-g)^{2}}{G}=\frac{G^{2} \beta}{2 w^{4}}(\Lambda+U) .
$$

By these expressions, we obtain (6.9).

We now show (a), (b) of (1). Let $p \neq 1$. Then $r$ is a periodic function with primitive period $2 K(p) / y$, which attains the maximum value precisely when $t=2 m K(p) / y$, and the minimum value precisely when $t=(2 m+1) K(p) / y$, where $m$ is an arbitrary integer. (There are no critical points other than the maximum and minimum points.) Next, we investigate the case $p=1$. Then, $S_{1}=0$. Also, it follows from (6.2) that $U-32 p^{2} w^{2}=-\beta Y_{2}^{2}-16 \leqslant 0$. Hence (6.3) implies $\xi=1$, and we have

$$
r(t)=\sqrt{\frac{U+\Lambda}{2 G \Lambda}} \operatorname{sech}(y t) .
$$

Therefore, $r$ attains the maximum value at $t=0$, and does not attain the minimum value. More precisely, $r$ is strictly monotone decreasing (resp. increasing) on $t>0$ (resp. $t<0$ ), and $\lim _{t \rightarrow \pm \infty} r(t)=0$.

By (6.9), the minimum and maximum values of $r$ are calculated as follows:

$$
r_{\min }=\sqrt{\frac{U-32 p^{2} w^{2}+\Lambda}{2 G \Lambda}}, \quad r_{\max }=\sqrt{\frac{U+\Lambda}{2 G \Lambda}} .
$$

Therefore, (6.3) completes the proof of (a). Also, the definition of $\Lambda$ completes the proof of (b).

Next, we show (2) and (3). By (6.19), it follows that

$$
\left(\frac{\partial}{\partial \theta}\right)_{\gamma(t)}=\frac{1}{2 f g}\left(g I_{+}-f I_{-}\right), \quad\left(\frac{\partial}{\partial \psi}\right)_{\gamma(t)}=\frac{1}{2 f g}\left(g I_{+}+f I_{-}\right) .
$$

Thus, if $r(t)>0$, then we have

$$
\begin{aligned}
\theta^{\prime}(t) & =\frac{\left\langle T,(\partial / \partial \theta)_{\gamma(t)}\right\rangle}{\left|(\partial / \partial \theta)_{\gamma(t)}\right|^{2}}=\frac{g\left\langle I_{+}, T\right\rangle-f\left\langle I_{-}, T\right\rangle}{2 f g r(t)^{2}} \\
& =\frac{-(f-g)}{8 G}+\frac{-2 G^{1 / 2} w^{4} D_{1}}{\beta\left[\Lambda+U-32 p^{2} w^{2} \operatorname{sn}^{2}(y t, p)\right]} .
\end{aligned}
$$

Thus, if $r(t)>0$ for all $t \in \boldsymbol{R}$, that is, if (6.10) does not hold or $p=1$, then $\theta(t)$ is expressed as (6.12).

Similarly, if $r(t)<1 / \sqrt{G}$, then we have

$$
\psi^{\prime}(t)=\frac{\left\langle T,(\partial / \partial \psi)_{\gamma(t)}\right\rangle}{\left|(\partial / \partial \psi)_{\gamma(t)}\right|^{2}}=\frac{-(f+g)}{8 G}+\frac{2 G^{1 / 2} w^{4} D_{2}}{\beta\left[\Lambda-U+32 p^{2} w^{2} \operatorname{sn}^{2}(y t, p)\right]} .
$$


If $r(t)<1 / \sqrt{G}$ for all $t \in \boldsymbol{R}$, that is, if (6.11) does not hold, then $\Lambda-U>0$, and so

$$
\psi^{\prime}(t)=\frac{-(f+g)}{8 G}+\left(\frac{G^{1 / 2} \beta^{1 / 2} C_{2}}{2 w}\right)\left(\frac{1}{1+\zeta^{2} \operatorname{sn}^{2}(y t, p)}\right) .
$$

Hence $\psi(t)$ is expressed as (6.14).

Next, we consider the case where $r_{\min }=0$ or $r_{\max }=1 / \sqrt{G}$. We investigate the behavior of $\theta(t)$ (resp. $\psi(t))$ around the points where $r(t)=0$ (resp. $r(t)=1 / \sqrt{G})$.

LEMMA 6.2. Set $t_{1}=(2 m+1) K(p) / y$ and $t_{2}=2 m K(p) / y$, where $m$ is an arbitrary integer.

(1) Suppose that (6.10) holds and $p \neq 1$. (Hence $r\left(t_{1}\right)=0$.) Then, both $\lim _{t \rightarrow t_{1}+0} \theta(t)$ and $\lim _{t \rightarrow t_{1}-0} \theta(t)$ exist, and

$$
\lim _{t \rightarrow t_{1}+0} \theta(t)-\lim _{t \rightarrow t_{1}-0} \theta(t)=\pi \quad(\bmod 2 \pi) .
$$

(2) Suppose that (6.11) holds. (Hence $r\left(t_{2}\right)=1 / \sqrt{G}$.) Then, both $\lim _{t \rightarrow t_{2}+0} \psi(t)$ and $\lim _{t \rightarrow t_{2}-0} \psi(t)$ exist, and

$$
\lim _{t \rightarrow t_{2}+0} \psi(t)-\lim _{t \rightarrow t_{2}-0} \psi(t)=\pi \quad(\bmod 2 \pi) .
$$

ProOF. We show (1). Note that $\left(x_{1}, x_{2}, \psi\right)$ is a local coordinate system on a neighborhood of $\gamma\left(t_{1}\right)$. Let $x_{1}(t), x_{2}(t), \psi(t)$ be the $x_{1}, x_{2}, \psi$ components of $\gamma(t)$. To prove (1), it suffices to show that $x_{1}^{\prime}(t)\left(\partial / \partial x_{1}\right)+x_{2}^{\prime}(t)\left(\partial / \partial x_{2}\right)$ does not vanish at $t=t_{1}$. Except at $t_{1}$, it follows that

$$
\left|x_{1}^{\prime} \frac{\partial}{\partial x_{1}}+x_{2}^{\prime} \frac{\partial}{\partial x_{2}}\right|=\left|r^{\prime} \frac{\partial}{\partial r}+\theta^{\prime} \frac{\partial}{\partial \theta}\right| \geqslant\left|r^{\prime} \frac{\partial}{\partial r}\right|=\frac{\left|r^{\prime}\right|}{\sqrt{G} \bar{r}} .
$$

Hence it is sufficient to verify that the right hand side of the above expression approaches a positive number as $t \rightarrow t_{1}$. The expression (6.9) together with the assumption (6.10) yields

$$
r(t)=\frac{4 p w}{G^{1 / 2} \Lambda^{1 / 2}}|\operatorname{cn}(y t, p)| .
$$

Thus, as $t \rightarrow t_{1}$.

$$
\left|r^{\prime}(t)\right|=\frac{2 \beta^{1 / 2} p}{\Lambda^{1 / 2}}|\operatorname{sn}(y t, p)| \operatorname{dn}(y t, p) \rightarrow \frac{2 \beta^{1 / 2} p\left(1-p^{2}\right)^{1 / 2}}{\Lambda^{1 / 2}}>0
$$

By using the local coordinate system $\left(x_{3}, x_{4}, \theta\right)$ around $\gamma\left(t_{2}\right)$, we can verify (2) in the same way as (1).

Suppose that (6.10) holds and $p \neq 1$, that is, $r_{\min }=0$. Then $\Lambda+U=32 p^{2} w^{2}$. Since $r(t)>0$ on the interval $(-K / y, K / y)$, we have

$$
\theta(t)=\int_{0}^{t} \theta^{\prime}(t) d t=\frac{-(f-g)}{8 G} t+\frac{-G^{1 / 2} w^{2} D_{1}}{16 \beta p^{2}} \int_{0}^{t} \frac{d t}{\mathrm{cn}^{2}(y t, p)}
$$

for $t \in(-K / y, K / y)$. If $D_{1} \neq 0$, then the right hand side of the above expression diverges as $t \rightarrow K / y-0$, which contradicts Lemma 6.2. Thus, $D_{1}=0$. Therefore, by Lemma 6.2, 
we have (6.13). Similarly, we see that if (6.11) holds, then $D_{2}=0$. Hence (6.15) follows. The proofs of (2) and (3) are completed.

We now consider Case 2. We first suppose that $0<r(t)<1 / \sqrt{G}$ for all $t \in \boldsymbol{R}$. Since

$$
T=\sigma \frac{\partial}{\partial \theta}+\rho \frac{\partial}{\partial \psi},
$$

we see $\langle T, \partial / \partial r\rangle=0$, and hence $r(t)$ is constant. Set $r(t)=r_{0}$. Then $\gamma$ lies on the Clifford torus $\left\{x \in S^{3} ; x_{1}^{2}+x_{2}^{2}=r_{0}^{2}\right\}$. We also see that if $r(t)=0$ (resp. $1 / \sqrt{G}$ ) for some $t$, then $r(t)=0$ (resp. $1 / \sqrt{G}$ ) for all $t$, and $\gamma$ is a geodesic. Thus, in what follows, we may assume that $0<r(t)<1 / \sqrt{G}$ for all $t \in \boldsymbol{R}$.

It follows from (6.24) that $\theta^{\prime}(t)=\sigma, \psi^{\prime}(t)=\rho$. Hence we obtain (6.18). By (6.24) and $|T|=1$, we have

$$
\left(\sigma^{2}-\rho^{2}\right) r_{0}^{2}+\rho^{2} / G=1 .
$$

Now, we show $\sigma^{2}-\rho^{2} \neq 0$. By (5.1), the squared curvature of $\gamma$ is expressed as

$$
\alpha_{3}=G r_{0}^{2} \bar{r}_{0}^{2}\left(\sigma^{2}-\rho^{2}\right)^{2} .
$$

Hence the assumption that $\gamma$ is not a geodesic implies $\sigma^{2}-\rho^{2} \neq 0$. Consequently, (6.25) yields (6.17).

Finally, we express the constants $\sigma, \rho$ by $\beta, \eta, w$. By (6.24) together with the assumption that the orientation of $(\partial / \partial r, \partial / \partial \theta, \partial / \partial \psi)$ is negative, we have

$$
N=\sqrt{G} \bar{r}_{0} \frac{\partial}{\partial r}, \quad B=-\frac{\rho \bar{r}_{0}}{r_{0}} \frac{\partial}{\partial \theta}+\frac{\sigma r_{0}}{\bar{r}_{0}} \frac{\partial}{\partial \psi},
$$

which implies that the torsion of $\gamma$ is given by

$$
\tau=\left\langle\nabla_{T} N, B\right\rangle=-\rho \sigma / \sqrt{G} .
$$

Substituting (6.17) to (6.26) yields

$$
\sigma^{2}+\rho^{2}=G \beta+\tau^{2}+G .
$$

Also, by (3.10), (6.4) and (6.5), the torsion is expressed by $\beta, \eta, w$ as

$$
\tau= \pm \sqrt{G \beta} \Omega \text {. }
$$

Therefore, by (6.27), (6.28), (6.29) and $\rho>|\sigma|$, we obtain (6.16).

We show that the $\partial / \partial r, \partial / \partial \theta, \partial / \partial \psi$ components of $M_{1}(t), M_{2}(t)$ are explicitly expressed. First, we express $M_{1}, M_{2}$ in terms of the Frenet frame.

LEMMA 6.3. If $p \neq w$ or $p=w=1$, then

$$
\begin{gathered}
M_{1}(t)=(\cos \varphi(t)) N(t)+(\sin \varphi(t)) B(t), \\
M_{2}(t)=-(\sin \varphi(t)) N(t)+(\cos \varphi(t)) B(t),
\end{gathered}
$$

where

$$
\varphi(t)= \pm\left[\frac{-V X}{w} \Pi\left(y t, q^{2}, p\right)+(1-v) \eta G^{1 / 2} \beta^{1 / 2} t\right]+\varphi(0) .
$$


If $p=w \neq 1$, then

$$
\begin{gathered}
M_{1}(t)=(\cos \varphi(t)) \hat{N}(t)+(\sin \varphi(t)) \hat{B}(t), \\
M_{2}(t)=-(\sin \varphi(t)) \hat{N}(t)+(\cos \varphi(t)) \hat{B}(t),
\end{gathered}
$$

where

$$
\varphi(t)= \pm(1-v) \eta G^{1 / 2} \beta^{1 / 2} t+\varphi(0),
$$

and $\hat{N}(t), \hat{B}(t)$ denote the generalized Frenet vectors defined by (3.17).

Proof. Let $p \neq w$ or $p=w=1$. Since the orientation of $\left(T, M_{1}, M_{2}\right)$ is positive, there exists a smooth function $\varphi(t)$ such that $M_{1}(t)$ and $M_{2}(t)$ are expressed as (6.30) and (6.31). By substituting (3.7) to (3.5), we have (6.32). Similarly, we get the expression of the case where $p=w \neq 1$.

Proposition 6.4. Suppose that $r(t) \neq 0,1 / \sqrt{G}$ and $k(t) \neq 0$. Then the $\partial / \partial r, \partial / \partial \theta$, $\partial / \partial \psi$ components of $M_{1}(t)$ and $M_{2}(t)$ are explicitly expressed in terms of Jacobi sn, cn and dn functions.

Proof. By Lemma 6.3, it suffices to seek for the explicit expressions of the $\partial / \partial r, \partial / \partial \theta$, $\partial / \partial \psi$ components of $N(t), B(t), \hat{N}(t)$ and $\hat{B}(t)$. We express them by $r(t), \theta^{\prime}(t), \psi^{\prime}(t)$ and their derivatives.

Substituting $T=r^{\prime}(\partial / \partial r)+\theta^{\prime}(\partial / \partial \theta)+\psi^{\prime}(\partial / \partial \psi)$ to $N=(1 / k) \nabla_{T} T$ yields

$$
N=h_{1} \frac{\partial}{\partial r}+h_{2} \frac{\partial}{\partial \theta}+h_{3} \frac{\partial}{\partial \psi},
$$

where

$$
\begin{aligned}
& h_{1}=\frac{1}{k}\left(r^{\prime \prime}+\frac{\left(r^{\prime}\right)^{2} r}{\bar{r}^{2}}+G r \bar{r}^{2}\left(\left(\psi^{\prime}\right)^{2}-\left(\theta^{\prime}\right)^{2}\right)\right), \\
& h_{2}=\frac{1}{k}\left(\theta^{\prime \prime}+\frac{2 \theta^{\prime} r^{\prime}}{r}\right), \quad h_{3}=\frac{1}{k}\left(\psi^{\prime \prime}-\frac{2 \psi^{\prime} r^{\prime} r}{\bar{r}^{2}}\right) .
\end{aligned}
$$

Also, by substituting (6.20) to $B=(1 / k)(H-2 v a T)$, we obtain

$$
B=\frac{1}{k}\left[-2 v a r^{\prime} \frac{\partial}{\partial r}+\left(\frac{f+g}{4 \sqrt{G}}-2 v a \theta^{\prime}\right) \frac{\partial}{\partial \theta}+\left(\frac{f-g}{4 \sqrt{G}}-2 v a \psi^{\prime}\right) \frac{\partial}{\partial \psi}\right] .
$$

Now, the explicit expressions of $r(t), \theta^{\prime}(t)$ and $\psi^{\prime}(t)$ in terms of Jacobi sn function are given by (6.9), (6.22) and (6.23). Hence $r^{\prime}, r^{\prime \prime}, \theta^{\prime \prime}, \psi^{\prime \prime}$ are explicitly expressed in terms of Jacobi sn, cn, dn functions. Consequently, the $\partial / \partial r, \partial / \partial \theta, \partial / \partial \psi$ components of $N(t), B(t)$ are also explicitly expressed in terms of Jacobi sn, cn, dn functions. Since $\hat{N}(t)=N(t)$ or $-N(t)$, this also holds for $\hat{N}$. Similarly, this holds for $\hat{B}$.

7. Closure conditions. We call $\{\gamma, M\}$ a closed Kirchhoff elastic rod if $\{\gamma, M\}$ is a Kirchhoff elastic rod and $\gamma$ is periodic. In this section, we investigate the condition that $\{\gamma, M\}$ be a closed Kirchhoff elastic rod in terms of $(\beta, \eta, p, w)$. 
We define two functions $\Delta \theta, \Delta \psi$ on $\tilde{\mathcal{P}}$ as follows. Let $\{\gamma, M\}$ be a Kirchhoff elastic rod in $S^{3}$ corresponding to $(\beta, \eta, p, w) \in \tilde{\mathcal{P}}$. We may assume that the constant $b\left(=k^{2}(\tau-v a)\right)$ for $\{\gamma, M\}$ is positive or zero. By Theorem 6.1, if $0<p<1$, then $r(t)$ is a $(2 K(p) / y)$ periodic function and $\theta^{\prime}(t), \psi^{\prime}(t)$ are $(2 K(p) / y)$-periodic or constant functions. We set

$$
\Delta \theta=\theta(2 K / y)-\theta(0), \quad \Delta \psi=\psi(K / y)-\psi(-K / y) .
$$

That is, $\Delta \theta$ and $\Delta \psi$ are the changes in $\theta(t)$ and $\psi(t)$, respectively, through the primitive period of $r$. By Theorem 6.1, $\Delta \theta$ and $\Delta \psi$ are expressed by $(\beta, \eta, p, w)$ as follows.

PROPOSITION 7.1.

$$
\begin{aligned}
& \Delta \theta=\left\{\begin{array}{l}
\frac{-(f-g) K(p)}{4 G y}+2 C_{1} \Pi\left(K(p), \xi^{2}, p\right) \quad \text { if }(6.10) \text { does not hold, } \\
\frac{-(f-g) K(p)}{4 G y}+\pi \quad \text { if }(6.10) \text { holds },
\end{array}\right. \\
& \Delta \psi=\left\{\begin{array}{l}
\frac{-(f+g) K(p)}{4 G y}+2 C_{2} \Pi\left(K(p),-\zeta^{2}, p\right) \quad \text { if }(6.11) \text { does not hold, } \\
\frac{-(f+g) K(p)}{4 G y}+\pi \quad \text { if }(6.11) \text { holds, }
\end{array}\right.
\end{aligned}
$$

where we take the upper sign in $f, g, C_{1}, C_{2}$.

We regard $\Delta \theta$ and $\Delta \psi$ as functions of $(\beta, \eta, p, w)(\in \tilde{\mathcal{P}})$ defined by the right hand sides of (7.2) and (7.3). We write them as $\Delta \theta(\beta, \eta, p, w)$ and $\Delta \psi(\beta, \eta, p, w)$. Then we have the following

THEOREM 7.2. Let $\{\gamma, M\}$ be a Kirchhoff elastic rod in $S^{3}$ corresponding to $(\beta, \eta, p, w) \in \tilde{\mathcal{P}}$. Then $\{\gamma, M\}$ is a closed Kirchhoff elastic rod if and only if either the following (1) or (2) holds, where $\boldsymbol{Q}$ denotes the set of all rational numbers.

(1) $0<p<1$ and $\Delta \theta(\beta, \eta, p, w) /(2 \pi), \Delta \psi(\beta, \eta, p, w) /(2 \pi) \in Q$.

(2) $\quad p=0$ and $\left(Q_{1}-Q_{2}\right) /\left(Q_{1}+Q_{2}\right) \in Q$.

PROOF. Without loss of generality, we may assume that the constant $b$ for $\{\gamma, M\}$ is positive or zero. Suppose that $\gamma$ is periodic. If $p=1$, then $k(t)$ is not periodic. Hence $\gamma$ is not periodic, too. Next, let $p=0$. Then $r(t)$ is constant, so that $\gamma$ lies on a Clifford torus. Also, $\theta(t)$ and $\psi(t)$ are expressed as (6.18). Thus, the periodicity of $\gamma$ implies that $\theta^{\prime}(t) / \psi^{\prime}(t)=\sigma / \rho$ is a rational number, and so is $\left(Q_{1}-Q_{2}\right) /\left(Q_{1}+Q_{2}\right)$. That is, (2) holds. Next, let $0<p<1$. Let $l$ denote the primitive period of $\gamma$. Then, since $k(t)$ is $(2 K / y)$ periodic, $l$ is expressed as $l=m(2 K / y)$, where $m$ is a non-zero integer. It then follows from (7.1) that $\theta(t+l)=\theta(t)+m \Delta \theta$ for all $t$ except at the points where $r(t)=0$. Hence, $m \Delta \theta(\beta, \eta, p, w) /(2 \pi)$ is an integer, so that $\Delta \theta(\beta, \eta, p, w) /(2 \pi) \in \boldsymbol{Q}$. Similarly, we see that $\Delta \psi(\beta, \eta, p, w) /(2 \pi) \in \boldsymbol{Q}$. Thus, (1) holds.

We show the converse. Suppose that (1) holds. Then there exists a non-zero integer $m$ such that $m \Delta \theta, m \Delta \psi \in 2 \pi \boldsymbol{Z}$. Thus, $\theta(t+m(2 K / y))=\theta(t) \bmod 2 \pi$ holds for all $t$ except at the points where $r(t)=0$. Also, $\psi(t+m(2 K / y))=\psi(t) \bmod 2 \pi$ holds for all $t$ except 
at the points where $r(t)=1 / \sqrt{G}$, while $r(t+m(2 K / y))=r(t)$ holds for all $t$. Hence we obtain that $\gamma(t+m(2 K / y))=\gamma(t)$ holds for all $t$, that is, $\gamma$ is periodic. Next, we suppose that (2) holds. Then $\gamma$ is a helix on a Clifford torus and $\theta^{\prime}(t) / \psi^{\prime}(t)$ is a rational number. Therefore, $\gamma$ is periodic.

8. Closed Kirchhoff elastic rods. In this section, by using Theorem 7.2 , we give an example of closed Kirchhoff elastic rods. Specifically, we give a smooth two-parameter family of closed Kirchhoff elastic rods including both helices and non-helices. Precisely, we show the following

THEOREM 8.1. There exists a smooth two-parameter family $\left\{\gamma^{\lambda, \omega}, M^{\lambda, \omega}\right\}(0 \leqslant \lambda \ll$ $1,|\omega| \ll 1)$ of closed Kirchhoff elastic rods satisfying the following. If $\lambda=0$, then $\gamma^{\lambda, \omega}$ is a helix, and if $\lambda \neq 0$, then $\gamma^{\lambda, \omega}$ is not a helix. Moreover, if $\lambda_{1}, \lambda_{2} \neq 0$ and $\left(\lambda_{1}, \omega_{1}\right) \neq\left(\lambda_{2}, \omega_{2}\right)$ hold, then $\gamma^{\lambda_{1}, \omega_{1}}$ and $\gamma^{\lambda_{2}, \omega_{2}}$ are not congruent.

To prove Theorem 8.1, we need three lemmas. We regard $\Delta \theta$ and $\Delta \psi$ as formal functions on

$$
\hat{\mathcal{P}}=\left\{(\beta, \eta, p, w) \in \boldsymbol{R}^{4} ; \beta>0,0 \leqslant|p| \leqslant w \leqslant 1, w>0\right\} .
$$

Note that $\Delta \theta, \Delta \psi$ are even functions in $p$. We calculate the expressions of $\Delta \theta, \Delta \psi$ evaluated at $p=0$. Let sgn denote the function on $\boldsymbol{R}$ defined by

$$
\operatorname{sgn} x= \begin{cases}1 & \text { if } x>0, \\ 0 & \text { if } x=0, \\ -1 & \text { if } x<0 .\end{cases}
$$

Lemma 8.2. Suppose that $p=0$. Then the three conditions (6.10), (6.11) and $\beta^{1 / 2} \Gamma= \pm 1$ are equivalent. If $\beta^{1 / 2} \Gamma \neq \pm 1$, then $\Delta \theta$ and $\Delta \psi$ are expressed as follows:

$$
\Delta \theta(\beta, \eta, 0, w)=\frac{\pi w}{\beta^{1 / 2}}\left(-\varepsilon_{1} Q_{1}+\varepsilon_{2} Q_{2}\right), \quad \Delta \psi(\beta, \eta, 0, w)=\frac{\pi w}{\beta^{1 / 2}}\left(-\varepsilon_{1} Q_{1}-\varepsilon_{2} Q_{2}\right),
$$

where

$$
\varepsilon_{1}=\operatorname{sgn}\left(\beta^{1 / 2} \Gamma+1\right), \quad \varepsilon_{2}=\operatorname{sgn}\left(-\beta^{1 / 2} \Gamma+1\right) .
$$

PROOF. A straightforward calculation yields the following.

$$
\begin{aligned}
& Y_{1}=\frac{4 w^{2}}{\beta}(\beta \Omega \Gamma-1), \quad R=2 w^{2} \Gamma, \quad Y_{2}=\frac{4 w^{2}}{\beta}\left(\beta \Gamma^{2}-1\right), \\
& U=\frac{16 w^{4}}{\beta}\left(\beta \Gamma^{2}-1\right)\left[1-\beta\left(\Omega^{2}+1\right)\right], \\
& \Lambda=\frac{16 w^{4}}{\beta}\left(1-\beta \Gamma^{2}\right) \varepsilon_{1} \varepsilon_{2} Q_{1} Q_{2}, \quad S_{1}=\frac{4 w^{3}}{\beta}\left(\beta \Gamma^{2}-1\right), \\
& \left\langle I_{+}, T\right\rangle=2 G\left(\beta^{1 / 2} \Omega-1\right)\left(\beta^{1 / 2} \Gamma+1\right), \\
& \left\langle I_{-}, T\right\rangle=2 G\left(-\beta^{1 / 2} \Omega-1\right)\left(-\beta^{1 / 2} \Gamma+1\right), \\
& f=2 G^{3 / 2}\left(\beta^{1 / 2} \Gamma+1\right) \varepsilon_{1} Q_{1}, \quad g=2 G^{3 / 2}\left(-\beta^{1 / 2} \Gamma+1\right) \varepsilon_{2} Q_{2} .
\end{aligned}
$$


Then it is easy to see that the three conditions (6.10), (6.11) and $\beta^{1 / 2} \Gamma= \pm 1$ are equivalent. Thus, if $\beta^{1 / 2} \Gamma \neq \pm 1$, then $\Lambda+U>|U|+U \geqslant 0, \Lambda-U>|U|-U \geqslant 0$, and hence $C_{1}$, $C_{2}, \xi, \zeta, \Delta \theta, \Delta \psi$ are well-defined.

Suppose that $\beta^{1 / 2} \Gamma \neq \pm 1$. By (6.21), we find $\Delta \theta$ to be

$$
\Delta \theta(\beta, \eta, 0, w)=\frac{2 K(0)}{y}\left(\frac{g\left\langle I_{+}, T\right\rangle-f\left\langle I_{-}, T\right\rangle}{2 f g(U+\Lambda) /(2 G \Lambda)}\right) .
$$

Substituting (8.1) to the expression above, we then have

$$
\begin{aligned}
\Delta \theta(\beta, \eta, 0, w) & =\frac{2 \pi w}{G^{1 / 2} \beta^{1 / 2}}\left(\frac{G^{1 / 2}\left[\left(\beta^{1 / 2} \Omega+1\right) \varepsilon_{1} Q_{1}+\left(\beta^{1 / 2} \Omega-1\right) \varepsilon_{2} Q_{2}\right]}{\left[\left(\Omega^{2}+1\right) \beta-1\right]+\varepsilon_{1} \varepsilon_{2} Q_{1} Q_{2}}\right) \\
& =\frac{\pi w}{\beta^{1 / 2}}\left(-\varepsilon_{1} Q_{1}+\varepsilon_{2} Q_{2}\right) .
\end{aligned}
$$

Similarly, we have the expression of $\Delta \psi(\beta, \eta, 0, w)$.

Let $\mathcal{S}$ denote the subset of $\hat{\mathcal{P}}$ defined by

$$
\mathcal{S}=\left\{(\beta, \eta, p, w) \in \hat{\mathcal{P}} ; p=0, \beta^{1 / 2} \Gamma \neq \pm 1, w \neq 1\right\} .
$$

The image of the map $(\Delta \theta /(2 \pi), \Delta \psi /(2 \pi)): \mathcal{S} \rightarrow \boldsymbol{R}^{2}$ is determined as follows. (We omit the proof.)

Lemma 8.3. Let $0<w_{0}<1$. Then,

$$
\begin{aligned}
&\left(\frac{\Delta \theta}{2 \pi}, \frac{\Delta \psi}{2 \pi}\right)\left(\left.\mathcal{S}\right|_{w=w_{0}}\right) \\
&=\left\{\left(v_{1}, v_{2}\right) \in \boldsymbol{R}^{2} ; v_{2}<0, v_{1}+v_{2}>1\right\} \\
& \bigcup\left\{\left(v_{1}, v_{2}\right) \in \boldsymbol{R}^{2} ; v_{1}+v_{2} \leqslant-w_{0}, v_{1}-v_{2}>1\right\} \\
& \bigcup\left\{\left(v_{1}, v_{2}\right) \in \boldsymbol{R}^{2} ; v_{2}<0, v_{1}-v_{2} \leqslant-w_{0}\right\} \\
& \bigcup\left\{\left(v_{1}, v_{2}\right) \in \boldsymbol{R}^{2} ; v_{2} \geqslant 0, v_{1}+v_{2} \leqslant-w_{0}, v_{1}-v_{2}>-1,\left(v_{1}, v_{2}\right) \neq\left(-w_{0}, 0\right)\right\} .
\end{aligned}
$$

We state the third lemma.

LEMMA 8.4. Let $\left(\beta_{0}, \eta_{0}, 0, w_{0}\right) \in \mathcal{S}$. Then $\Delta \theta$ and $\Delta \psi$ are smooth functions on a neighborhood of $\left(\beta_{0}, \eta_{0}, 0, w_{0}\right)$ in $\hat{\mathcal{P}}$, and

$$
\left.\frac{D(\Delta \theta, \Delta \psi)}{D(\beta, \eta)}\right|_{\left(\beta_{0}, \eta_{0}, 0, w_{0}\right)} \neq 0,
$$

where the left hand side denotes the Jacobian of the map $(\Delta \theta, \Delta \psi)$ at $\left(\beta_{0}, \eta_{0}, 0, w_{0}\right)$.

Proof. Since both (6.10) and (6.11) do not hold on a neighborhood of $\left(\beta_{0}, \eta_{0}, 0, w_{0}\right)$ in $\hat{\mathcal{P}}, \Delta \theta$ and $\Delta \psi$ are given by the upper expressions of the right hand sides of (7.2) and (7.3). Also, since $\beta, V, X, \Lambda, f$ and $g$ are positive at $\left(\beta_{0}, \eta_{0}, 0, w_{0}\right)$, we see that $\Delta \theta$ and $\Delta \psi$ are smooth on a neighborhood of $\left(\beta_{0}, \eta_{0}, 0, w_{0}\right)$ in $\hat{\mathcal{P}}$. We show (8.2). By Lemma 8.2, we have

$$
\left(\left.\Delta \theta\right|_{p=0}\right)^{2}+\left(\left.\Delta \psi\right|_{p=0}\right)^{2}=4 \pi^{2} w^{2}\left(1+\Omega^{2}+1 / \beta\right),
$$




$$
\left(\left.\Delta \theta\right|_{p=0}\right)\left(\left.\Delta \psi\right|_{p=0}\right)=-\frac{4 \pi^{2} w^{2} \Omega}{\beta^{1 / 2}} .
$$

We write $\left.\Delta \theta\right|_{p=0},\left.\Delta \psi\right|_{p=0}$ as $\Delta \theta, \Delta \psi$. Differentiating (8.3) and (8.4) with respect to $\beta$ and $\eta$ yields

$$
\left(\begin{array}{cc}
\Delta \theta & \Delta \psi \\
\Delta \psi & \Delta \theta
\end{array}\right)\left(\begin{array}{cc}
\frac{\partial \Delta \theta}{\partial \beta} & \frac{\partial \Delta \theta}{\partial \eta} \\
\frac{\partial \Delta \psi}{\partial \beta} & \frac{\partial \Delta \psi}{\partial \eta}
\end{array}\right)=2 \pi^{2} w^{2}\left(\begin{array}{cc}
-1 / \beta^{2} & 2 \nu \Omega \\
-\Omega / \beta^{1 / 2} & -2 \nu / \beta^{1 / 2}
\end{array}\right) .
$$

By calculating the determinants of the both sides, we have

$$
\left[(\Delta \theta)^{2}-(\Delta \psi)^{2}\right] \frac{D(\Delta \theta, \Delta \psi)}{D(\beta, \eta)}=\frac{8 \pi^{4} \nu w^{4}}{\beta^{5 / 2}}\left(1+\beta^{2} \Omega^{2}\right),
$$

where the right hand side does not vanish. Hence we obtain (8.2).

Proof of THEOREM 8.1. By Lemma 8.3, there exists a rational point in the set $(\Delta \theta /(2 \pi), \Delta \psi /(2 \pi))(\mathcal{S})$. Let $\left(\beta_{0}, \eta_{0}, 0, w_{0}\right)$ be an element of $\mathcal{S}$ such that

$$
\left(\frac{\Delta \theta}{2 \pi}, \frac{\Delta \psi}{2 \pi}\right)\left(\beta_{0}, \eta_{0}, 0, w_{0}\right) \in \boldsymbol{Q} \times \boldsymbol{Q} .
$$

By Lemma 8.4 and the implicit function theorem together with the fact that $\Delta \theta$ and $\Delta \psi$ are even in $p$, there exists smooth functions $\beta(p, w)$ and $\eta(p, w)$ on a neighborhood of $\left(0, w_{0}\right)$ such that $\beta\left(0, w_{0}\right)=\beta_{0}, \eta\left(0, w_{0}\right)=\eta_{0}$ and

$$
\begin{aligned}
& \beta(p, w)=\beta(-p, w), \quad \eta(p, w)=\eta(-p, w), \\
& \Delta \theta(\beta(p, w), \eta(p, w), p, w)=\Delta \theta\left(\beta_{0}, \eta_{0}, 0, w_{0}\right), \\
& \Delta \psi(\beta(p, w), \eta(p, w), p, w)=\Delta \psi\left(\beta_{0}, \eta_{0}, 0, w_{0}\right) .
\end{aligned}
$$

Let $\left\{\gamma^{p, w}, M^{p, w}\right\}$ be the curve with adapted orthonormal frame obtained by substituting $(\beta, \eta)=(\beta(p, w), \eta(p, w))$ to (6.9), (6.12), (6.14) and (6.32) with $\theta(0)=0, \psi(0)=0$ and $\varphi(0)=0$. Then, for each $(p, w)$ satisfying $p \neq 0,\left\{\gamma^{p, w}, M^{p, w}\right\}$ is a Kirchhoff elastic rod corresponding to $(\beta(p, w), \eta(p, w),|p|, w) \in \tilde{\mathcal{P}}$. Note that $\left\{\gamma^{p, w}, M^{p, w}\right\}=$ $\left\{\gamma^{-p, w}, M^{-p, w}\right\}$ holds.

Let $p>0$. We show that $\gamma^{p, w}$ is periodic. By the definitions of $\beta(p, w)$ and $\eta(p, w)$, together with the assumption (8.5),

$$
\Delta \theta(\beta(p, w), \eta(p, w), p, w) / 2 \pi=\Delta \theta\left(\beta_{0}, \eta_{0}, 0, w_{0}\right) / 2 \pi \in \boldsymbol{Q} .
$$

Similarly, $\Delta \psi(\beta(p, w), \eta(p, w), p, w) / 2 \pi \in \boldsymbol{Q}$. Therefore, by Theorem 7.2 , we have that $\gamma^{p, w}$ is periodic. Since $p \neq 0, \gamma^{p, w}$ is not a helix.

Next, let $p=0$. We first show that $\left\{\gamma^{0, w}, M^{0, w}\right\}$ is a Kirchhoff elastic rod whose centerline is a helix. Since $r(0, w, t),(d \theta / d t)(0, w, t)$ and $(d \psi / d t)(0, w, t)$ are constant in $t$ and $0<r(0, w, t)<1 / \sqrt{G}$, we see that $\gamma^{0, w}$ is a helix. Also, the continuity of $\left|d \gamma^{p, w} / d t\right|$ implies that $\gamma^{0, w}$ is a unit-speed curve. Since $\varphi(0, w, t)$ is an affine linear function in $t$, we 
have that $\left\{\gamma^{0, w}, M^{0, w}\right\}$ is a Kirchhoff elastic rod. We show that $\gamma^{0, w}$ is periodic. Since $\gamma^{0, w}$ is unit-speed, $\theta^{\prime} \neq 0$ or $\psi^{\prime} \neq 0$. First, let $\theta^{\prime} \neq 0$. By (8.5), we have

$$
\frac{\psi^{\prime}}{\theta^{\prime}}=\frac{\Delta \psi(\beta(0, w), \eta(0, w), 0, w)}{\Delta \theta(\beta(0, w), \eta(0, w), 0, w)}=\frac{\Delta \psi\left(\beta_{0}, \eta_{0}, 0, w_{0}\right) /(2 \pi)}{\Delta \theta\left(\beta_{0}, \eta_{0}, 0, w_{0}\right) /(2 \pi)} \in \boldsymbol{Q} .
$$

Hence $\gamma^{0, w}$ is periodic. When $\psi^{\prime} \neq 0$ as well, we see that $\gamma^{0, w}$ is periodic in the same way as above.

We show that if $p_{1}, p_{2}>0$ and $\left(p_{1}, w_{1}\right) \neq\left(p_{2}, w_{2}\right)$ hold, then $\gamma^{p_{1}, w_{1}}$ and $\gamma^{p_{2}, w_{2}}$ are not congruent. Seeking a contradiction, we suppose that $\gamma^{p_{1}, w_{1}}$ and $\gamma^{p_{2}, w_{2}}$ are congruent. Denote the parameters $\beta, y$, etc. for $\left\{\gamma^{p_{i}, w_{i}}, M^{p_{i}, w_{i}}\right\}$ by $\beta_{i}, y_{i}$, etc., where $i=1$ or 2 . Since $p_{1}, p_{2}>0$ and the squared curvature of $\gamma^{p_{1}, w_{1}}$ coincides with that of $\gamma^{p_{2}, w_{2}}$, we have $\beta_{1}=\beta_{2}, p_{1} / w_{1}=p_{2} / w_{2}$ and $\operatorname{sn}\left(y_{1} t, p_{1}\right)=\operatorname{sn}\left(y_{2} t, p_{2}\right)$ for all $t$. By calculating the derivatives of the both sides of the above equation at $t=0$, we obtain $y_{1}=y_{2}$, which implies $w_{1}=w_{2}$, and hence $p_{1}=p_{2}$. This contradicts the assumption.

We set $\lambda=p, \omega=w-w_{0}$, and rewrite $\left\{\gamma^{\lambda, w_{0}+\omega}, M^{\lambda, w_{0}+\omega}\right\}$ as $\left\{\gamma^{\lambda, \omega}, M^{\lambda, \omega}\right\}$. Then the smooth two-parameter family $\left\{\gamma^{\lambda, \omega}, M^{\lambda, \omega}\right\}(0 \leqslant \lambda \ll 1,|\omega| \ll 1)$ of closed Kirchhoff elastic rods satisfies the properties in Theorem 8.1 .

\section{REFERENCES}

[1] M. Barros, O. Garay And D. Singer, Elasticae with constant slant in the complex projective plane and new examples of Willmore tori in five spheres, Tohoku Math. J. 51 (1999), 177-192.

[2] R. BRYANT AND P. GRIFFITHS, Reduction for constrained variational problems and $\int k^{2} / 2 d s$, Amer. J. Math. 108 (1986), 525-570.

[ 3 ] P. F. BYRD AND M. D. FRIEDMAN, Handbook of elliptic integrals for engineers and physicists, Springer, Berlin, 1954.

[4] P. GRIFFITHS, Exterior differential systems and the calculus of variations, Birkhäuser, Boston, 1983.

[ 5 ] H. Hasimoto, Motion of a vortex filament and its relation to elastica, J. Phys. Soc. Japan 31 (1971), 293-294.

[ 6] H. Hasimoto, A soliton on a vortex filament, J. Fluid Mech. 51 (1972), 477-485.

[7] T. IVEY AND D. Singer, Knot types, homotopies and stability of closed elastic rods, Proc. London Math. Soc. (3) 79 (1999), 429-450.

[ 8 ] V. JurdjeVIC, Non-Euclidean elastica, Amer. J. Math. 117 (1995), 93-124.

[ 9 ] S. KaWAKUbo, Stability and bifurcation of circular Kirchhoff elastic rods, Osaka J. Math. 37 (2000), 93-137, Errata, Osaka J. Math. 37 (2000), 525.

[10] S. KawaKubo, Kirchhoff elastic rods in a Riemannian manifold, Tohoku Math. J. 54 (2002), 179-193.

[11] S. KIDA, A vortex filament moving without change of form, J. Fluid Mech. 112 (1981), 397-409.

[12] S. Kobayashi And K. Nomizu, Foundations of differential geometry, I, II, Interscience, 1963, 1969.

[13] N. KoIso, Elasticae in a Riemannian submanifold, Osaka J. Math. 29 (1992), 539-543.

[14] N. KoIso, The vortex filament equation and a semilinear Schrödinger equation in a Hermitian symmetric space, Osaka J. Math. 34 (1997), 199-214

[15] J. LAnger And D. Singer, Knotted elastic curves in $\boldsymbol{R}^{3}$, J. London Math. Soc. (2) 30 (1984), 512-520.

[16] J. LANGer AND D. Singer, Curves in the hyperbolic plane and mean curvature of tori in 3-space, Bull. London Math. Soc. 16 (1984), 531-534.

[17] J. LANGER AND D. Singer, The total squared curvature of closed curves, J. Differential Geom. 20 (1984), $1-22$ 
[18] J. LANGer AND D. Singer, Curve-straightening in Riemannian manifolds, Ann. Global Anal. Geom. 5 (1987), 133-150.

[19] J. LANGER AND D. Singer, Liouville integrability of geometric variational problems, Comment. Math. Helv. 69 (1994), 272-280.

[20] J. LANGER AND D. Singer, Lagrangian aspects of the Kirchhoff elastic rod, SIAM Rev. 38 (1996), 605-618.

[21] U. PINKaLL, Hopf tori in $S^{3}$, Invent. Math. 81 (1985), 379-386.

[22] Y. SHI AND J. HEARST, The Kirchhoff elastic rod, the nonlinear Schrödinger equation, and DNA supercoiling, J. Chem. Phys. 101 (1994), 5186-5200.

Department of Applied Mathematics

FUKUOKA UNIVERSITY

FUKUOKA 814-0180

JAPAN

E-mail address: kawakubo@math.sci.fukuoka-u.ac.jp 